\title{
High-order maximum-entropy collocation methods ${ }^{1}$
}

\author{
F. Greco ${ }^{\mathrm{a}, *}$, M. Arroyo ${ }^{\mathrm{a}}$ \\ ${ }^{a}$ Laboratori de Càlcul Numèric, Universitat Politècnica de Catalunya-BarcelonaTech, Jordi Girona 1, E-08034 \\ Barcelona, Spain.
}

\begin{abstract}
This paper considers the approximation of partial differential equations with a point collocation framework based on high-order local maximum-entropy schemes (HOLMES). In this approach, smooth basis functions are computed through an optimization procedure and the strong form of the problem is directly imposed at the collocation points, reducing significantly the computational times with respect to the Galerkin formulation. Furthermore, such a method is truly meshfree, since no background integration grid is necessary. The validity of HOLMES collocation is verified with supportive numerical examples, where the expected convergence rates are obtained. This includes the approximation of PDEs on domains bounded by implicit and explicit (NURBS) curves, illustrating a direct integration between geometric modeling and numerical analysis.
\end{abstract}

Keywords: HOLMES; maximum-entropy; meshfree; high-order; collocation; NURBS;

\section{Introduction}

The numerical approximation of partial differential equations (PDEs) is a crucial task in many fields of science and engineering. Over the last years, the most widely used methodology has been the finite element method (FEM), based on the Galerkin method and the weak form of the PDEs, which is compatible with $C^{0}$ basis functions. However, if an approximation of the solution is expressed as a linear combination of smoother basis functions, then collocation approaches are also possible, which consist in the imposition of the strong form of the governing equations in a set of isolated points called collocation points. Since no numerical integration is involved, this can introduce several computational and implementation advantages.

\footnotetext{
${ }^{1}$ C) 2020. This manuscript version is made available under the CC-BY-NC-ND 4.0 license http://creativecommons.org/licenses/by-nc-nd/4.0/.

Published version available at https://doi.org/10.1016/j.cma.2020.113115.

${ }^{*}$ Correspondence to: francesco.greco@upc.edu
} 
A number of approximation methods rely on smooth basis functions and are then compatible with collocation. A notable example is isogeometric analysis (IGA) [1] whose key idea is to employ spline functions both for the geometry representation and for the numerical analysis. These functions possess a higher inter-element continuity than FEM, which makes them suitable for collocation methods $[2,3,4]$. IGA collocation has indeed been applied to a variety of fields $[5,6,7,8,9]$ and ongoing research on the topic is very promising $[10,11]$. However, the tensor product structure of B-splines and NURBS (non-uniform rational B-splines) basis functions introduces an intrinsic limitation in volume meshing and local refinement, which can be only partially avoided with alternative spline functions $[12,13]$.

Another family of approximation schemes with smooth basis functions are meshfree (or meshless) methods $[14,15,16,17]$. As the name suggests, these schemes do not rely on a mesh or a structured grid to construct the numerical approximation, which makes them particularly suited for problems involving large deformations. Thanks to the high continuity of their basis functions, most meshfree methods have been used both in Galerkin and collocation approaches. Nodally integrated formulations have also been developed as an alternative integration strategy [18].

In the context of meshfree collocation, many methodologies have been successfully applied to different types of problems and an exhaustive list is beyond the scope of this paper. The Smooth Particle Hydrodynamics [19] can be considered as the first meshfree method and is based on collocation. Other schemes that have been used with collocation are Multiquadrics least square methods [20], the Reproducing Kernel Particle Method [21, 22], the Radial Basis Functions [23, 24] and the Finite Point Method [25]. The interested reader is referred to [26] and to [27] for a more detailed classification.

This paper focuses on the development of a collocation framework based on maximum-entropy (max-ent) basis functions. Max-ent methods were firstly introduced in computational mechanics to compute polygonal interpolants in [28] and then they were extended to meshfree approximation schemes in [29]. The underlying idea behind these methods is to consider an equivalence between basis functions and probability distributions and to maximize an entropy functional in order to compute the most unbiased basis functions, subject to reproducibility constraints. Obtained through an optimization procedure, these functions are able to reproduce constant and linear fields.

Since these pioneering works, it was noted that in order to impose higher order consistency 
conditions, the reproducibility constraints need to be carefully designed for feasibility. This allowed the definition of quadratic maximum entropy schemes with non-negative basis functions [30, 31] and also the definition of high-order local maximum entropy schemes (HOLMES) with signed functions [32]. In these schemes, although the positivity of the basis functions is lost, their $C^{\infty}$ continuity is preserved and an excellent accuracy is obtained in different applications, such as Helmholtz and shell problems [32]. Remarkably, even if the evaluation of the basis functions requires the iterative solution of a nonlinear optimization problem, the computational times are comparable to those required for other meshfree methods. In addition, signed basis functions are needed for high-order reproducibility conditions also for other methods, although the negative part can be reduced to some extent [33].

Max-ent methods have been successfully applied to a variety of fields using the standard Galerkin formulation with a background integration mesh $[34,35,36,37,38]$ but only a limited amount of research has been done on their use in a collocation framework [39, 40, 41]. This previous literature on max-ent collocation is based on the linear consistent schemes developed in [29]. However, examination of the literature on meshfree collocation methods suggests that at least quadratic consistency of the basis functions $[42,43]$ is required for the method to converge in second-order partial differential equations. If $n$ is the order of the basis functions, this gives an order of convergence of $n$ for even orders and $n-1$ for odd orders, which also follows the analogous IGA results [2]. Starting from this consideration, this work focuses on the development of a collocation framework based on HOLMES approximants, which provide arbitrary orders of consistency, are smooth and locally refineable.

In addition, this work explores the application of the collocation approach to PDEs on domains whose boundary has a smooth geometric definition, given either by an implicit description or by a NURBS curve. This second case is particularly relevant because it allows a tight integration between CAD information and numerical analysis. Furthermore, in contrast to IGA collocation, arbitrary shapes can be easily discretized with the proposed meshfree collocation method - see [44] for related work based on exponential basis functions.

The outline of the paper is the following: in Section 2 the point collocation framework is described and its advantages with respect to the Galerkin formulation are discussed; in Section 3 the HOLMES approximants are introduced and their properties are briefly discussed; then the 
accuracy of the proposed methodology is proved with supportive numerical examples in Section 4; finally, in Section 5, concluding remarks are given and possible future developments are discussed.

\section{Formulation of the point collocation framework}

Given a computational domain $\Omega$ with boundary $\Gamma$, the strong form of a boundary value problem can be expressed as

$$
\begin{cases}\mathcal{L} \mathbf{u}+\mathbf{b}=\mathbf{0} & \text { in } \Omega \\ \mathcal{B}^{g} \mathbf{u}=\mathbf{g} & \text { on } \Gamma^{g} \\ \mathcal{B}^{t} \mathbf{u}=\mathbf{t} & \text { on } \Gamma^{t},\end{cases}
$$

where $\mathbf{u}=\mathbf{u}(\mathbf{x})$ is the unknown field, $\mathcal{L}$ is a differential operator in $\Omega, \mathcal{B}^{g}$ is an operator on the Dirichlet part of the boundary $\Gamma^{g}, \mathcal{B}^{t}$ is a differential operator on the Neumann part of the boundary $\Gamma^{t}, \mathbf{b}$ is a source term and $\mathbf{g}$ and $\mathbf{t}$ are the imposed boundary conditions.

In contrast to the Galerkin formulation, in the point collocation framework the strong form of the problem is directly enforced in a set of points, called collocation points, and the solution $\mathbf{u}(\mathbf{x})$ is approximated as a linear combination of basis functions. Different strategies are possible for the choice of the collocation points and have been considered in the literature of both isogeometric and meshfree collocation methods. In the former case, it was shown that using suitable coordinates for the collocation points and using over-determined systems of equations leads to increased orders of convergence, equal to those of the Galerkin formulation [10,11]. Given the tensor product structure of IGA basis functions, these suitable coordinates can be easily identified in any dimension. The situation is different for meshfree formulations applied to unstructured points distributions, where the most natural choice for the collocation points are the nodes, which are adopted in this work. We do not pursue here the identification of optimal or privileged sets of collocations points because of the unstructured nature of the discretization that characterizes meshfree methods.

For a vectorial field $\mathbf{u}(\mathbf{x}): \Omega \rightarrow \Re^{d}$, the numerical approximation is expressed as

$$
\mathbf{u}^{h}(\mathbf{x})=\sum_{a=1}^{m} \phi_{a}(\mathbf{x}) \mathbf{u}_{a}
$$

where $\phi_{a}(\mathbf{x})$ is the basis function associated with the node $\mathbf{x}_{a}(a=1,2, \ldots, m)$ and $\mathbf{u}_{a}$ is a vector of coefficients. Using this approach and enforcing the strong form equation at the nodal coordinates, 
a linear system is obtained:

$$
\mathbf{K d}=\mathbf{f}
$$

where $\mathbf{K}, \mathbf{d}$ and $\mathbf{f}$ are often called stiffness matrix, displacement and force vector, although they have another meaning with respect to the Galerkin formulation. Their expression is given by:

$$
\begin{gathered}
\mathbf{K}=\left[\begin{array}{c}
\mathbf{K}^{i} \\
\mathbf{K}^{g} \\
\mathbf{K}^{t}
\end{array}\right], \quad \mathbf{f}=\left[\begin{array}{c}
\mathbf{f}^{i} \\
\mathbf{f}^{g} \\
\mathbf{f}^{t}
\end{array}\right] \\
\left\{\begin{array}{l}
\mathbf{K}_{a b}^{i}=\mathcal{L}\left[\phi_{b}\left(\mathbf{x}_{a}\right)\right], \quad \mathbf{f}_{a}^{i}=\mathbf{b}\left(\mathbf{x}_{a}\right) \\
\mathbf{K}_{a b}^{g}=\mathcal{B}^{g}\left[\phi_{b}\left(\mathbf{x}_{a}\right)\right], \quad \mathbf{f}_{a}^{g}=\mathbf{g}\left(\mathbf{x}_{a}\right) \\
\mathbf{K}_{a b}^{t}=\mathcal{B}^{t}\left[\phi_{b}\left(\mathbf{x}_{a}\right)\right], \quad \mathbf{f}_{a}^{t}=\mathbf{t}\left(\mathbf{x}_{a}\right)
\end{array}\right.
\end{gathered}
$$

and the numerical implementation is simple and straightforward.

\subsection{Computational advantages with respect to the Galerkin formulation}

Given the large support of max-ent basis functions, the point collocation framework introduces significant computational advantages with respect to the Galerkin formulation, which can be summarized by the two following points:

- the basis functions are evaluated in a much smaller number of points;

- the stiffness matrix filling procedure is straightforward with no memory bottlenecks;

The first point results from the fact that the number of quadrature points in the Galerkin formulation is in general higher than the number of nodes. Table 1 summarizes the expected ratios of quadrature points to nodes for the integration rules of $1^{\text {st }}$ to $5^{\text {th }}$ order given in [45], calculated for infinite regular triangular and tetrahedral meshes. Although the optimal number of quadrature points required for the weak form integration depends on the specific application and has not been studied in detail yet, it can be assumed that higher order basis functions require integration rules of higher polynomial order. This may lead for instance to a ratio of 84 quadrature points per node when using a $4^{\text {th }}$ order rule in three dimensions. In addition, the Galerkin formulation requires 
in each point the multiplication of the basis functions derivatives of each pair of neighbors to the point, whose number is in the order of several hundred in three dimensions.

While the evaluation of the contribution of each integration point is by itself very computationally demanding, in general, this part is not the bottleneck in Galerkin implementations. In fact, when dealing with large scale problems and sparse matrix implementations, the matrix filling procedure becomes particularly challenging due to memory bottlenecks [46]. Instead, in collocation methods the basis functions are evaluated at each collocation point and the matrix is directly filled row by row. Based on these considerations, a quantitative comparison of the computational times through numerical examples should strongly depend on the details of the Galerkin implementation and is beyond the scope of this work.

We finally note that, as it has been argued in the context of IGA, the bandwidth of the collocation matrix is smaller than that of the Galerkin matrix $[2,3]$.

Table 1: Number of quadrature points for Galerkin implementations of max-ent methods integrated over triangles (left) and tetrahedra (right).

$2 \mathrm{D}$

\begin{tabular}{|c|c|c|c|c|c|}
\hline Order & 1 & 2 & 3 & 4 & 5 \\
\hline Points per triangle & 1 & 3 & 6 & 6 & 7 \\
\hline Points per node & 2 & 6 & 12 & 12 & 14 \\
\hline
\end{tabular}

$3 \mathrm{D}$

\begin{tabular}{|c|c|c|c|c|c|}
\hline Order & 1 & 2 & 3 & 4 & 5 \\
\hline Points per tetrahedron & 1 & 4 & 8 & 14 & 14 \\
\hline Points per node & 6 & 24 & 48 & 84 & 84 \\
\hline
\end{tabular}

\section{The HOLMES approximants}

Maximum entropy basis functions were firstly developed by Sukumar [28], who was inspired by the max-ent formalism introduced by Jaynes in statistical mechanics [47]. In this approach, an entropy functional that depends on a discrete probability measure $\left\{p_{a}\right\}_{a=1}^{m}$ is maximized, subject to linear constraints on $p_{a}$. On noting the correspondence between basis functions $\left\{\phi_{a}\right\}_{a=1}^{m}$ and discrete probability measures $\left\{p_{a}\right\}_{a=1}^{m}$, the max-ent formalism was adopted for the construction of polygonal interpolants [28]. An extension to meshfree approximation schemes was introduced with the local maximum-entropy (LME) basis functions, developed by Arroyo and Ortiz in [29]. The main idea of LME schemes is to consider a Pareto optimum between the maximization of the entropy functional and the locality of the basis functions. Given a set of distinct nodes in $\Re^{d}$, 
located at $\mathbf{x}_{a}$, and being $\Omega=\operatorname{con}\left(\mathbf{x}_{1}, \ldots, \mathbf{x}_{m}\right) \subset \Re^{d}$ the convex hull of the nodal set, the associated formulation is to find $\mathbf{x} \mapsto \phi(\mathbf{x}): \Omega \rightarrow \Re_{+}^{m}$ as the solution of the following optimization problem:

$$
\min _{\phi \in \Re_{+}^{m}} \sum_{a=1}^{m} \phi_{a}(\mathbf{x}) \ln \phi_{a}(\mathbf{x})+\beta \sum_{a=1}^{m} \phi_{a}(\mathbf{x})\left|\mathbf{x}-\mathbf{x}_{a}\right|^{2},
$$

subject to the linear reproducing conditions:

$$
\begin{aligned}
\sum_{a=1}^{m} \phi_{a}(\mathbf{x}) & =1 \\
\sum_{a=1}^{m} \phi_{a}(\mathbf{x})\left(\mathbf{x}_{a}-\mathbf{x}\right) & =\mathbf{0}
\end{aligned}
$$

where $\Re_{+}^{m}$ is the non-negative orthant. In this formulation, the shape and the locality of the basis functions is controlled by the Pareto parameter $\beta$, which is normally replaced by a dimensionless parameter $\gamma=\beta h^{2}$, being $h$ a measure of the node spacing. In practice, LME schemes correspond to the polygonal interpolants of [28] for $\gamma=0$, while it can be proved that they recover linear Delaunay FEM when $\gamma \rightarrow \infty$.

The computation of this type of approximants with high-order consistency conditions is not straightforward, since adding the corresponding constraints to the ones in Eq. (6) may lead to an unfeasible problem, even for quadratic constraints [29]. This motivated several attempts to develop quadratic max-ent schemes, that include enrichment [48] following the partition-of-unity framework [49], combination of the max-ent formalism with de Boor's algorithm [50] and relaxation of the constraints. In this latter framework, [30] and [31] propose feasible quadratic constraints whereas in [32] the positivity constraints are released to formulate the HOLMES approximants, following an idea previously suggested in [51].

In the HOLMES formalism, the basis functions $\phi_{a}(\mathbf{x})$ are expressed as the difference between two non-negative contributions

$$
\phi_{a}(\mathbf{x})=\phi_{a}^{+}(\mathbf{x})-\phi_{a}^{-}(\mathbf{x})
$$

The entropy of $\phi_{a}$ cannot be computed since the logarithm is only defined on $\Re^{+}$. However, a modified entropy functional is defined as:

$$
H\left(\boldsymbol{\phi}^{+}, \boldsymbol{\phi}^{-}\right)=-\sum_{a=1}^{m} \phi_{a}^{+}(\mathbf{x}) \ln \phi_{a}^{+}(\mathbf{x})-\sum_{a=1}^{m} \phi_{a}^{-}(\mathbf{x}) \ln \phi_{a}^{-}(\mathbf{x}),
$$

which is no longer the entropy of a probability distribution, like the one used in [28]. However, on using such a functional, problem (5) is generalized to arbitrary orders of consistency $n$ in the 
following way:

$$
\begin{array}{r}
\min _{\phi^{+}, \phi^{-} \in \Re_{+}^{m}} \sum_{a=1}^{m} \phi_{a}^{+}(\mathbf{x}) \ln \phi_{a}^{+}(\mathbf{x})+\sum_{a=1}^{m} \phi_{a}^{-}(\mathbf{x}) \ln \phi_{a}^{-}(\mathbf{x})+ \\
+\frac{\gamma}{h^{p}} \sum_{a=1}^{m}\left(\phi_{a}^{+}(\mathbf{x})-\phi_{a}^{-}(\mathbf{x})\right)\left|\mathbf{x}-\mathbf{x}_{a}\right|_{p}^{p}
\end{array}
$$

subject to

$$
\begin{aligned}
\sum_{a=1}^{m}\left(\phi_{a}^{+}(\mathbf{x})-\phi_{a}^{-}(\mathbf{x})\right) & =1 \\
\sum_{a=1}^{m}\left(\phi_{a}^{+}(\mathbf{x})-\phi_{a}^{-}(\mathbf{x})\right)\left(\mathbf{x}_{a}-\mathbf{x}\right)^{\boldsymbol{\alpha}} & =0, \quad \forall \boldsymbol{\alpha} \in \mathbb{N}^{d}, 1 \leq|\boldsymbol{\alpha}| \leq n .
\end{aligned}
$$

In the equation above, the multi-index notation adopted in [32] is followed, where $\boldsymbol{\alpha}$ is a vector in $\mathbb{N}^{d}$ and $|\boldsymbol{\alpha}|=\sum_{i=1}^{d} \alpha_{i}$ is the order of $\boldsymbol{\alpha}$. At the same time, for a given point $\tilde{\mathbf{x}} \in \mathbb{R}^{d}$, the expression $\tilde{\mathbf{x}}^{\alpha}$ denotes the product $\Pi_{i=1}^{d} \tilde{x}_{i}^{\alpha_{i}}$.

In the definition of problem (9), a $p$-norm $|\mathbf{x}|_{p}=\left(\sum_{i=1}^{d}|x|_{i}^{p}\right)^{1 / p}$ is used, which generalizes the quadratic norm employed in Eq. (5) for LME schemes. In fact, in the context of Galerkin formulations, a sufficient condition for the convergence of HOLMES is $p>n$, which ensures that the basis functions have a proper exponential decay [32, 52]. Hence, this sufficient condition suggests values of $p>2$ for higher-order max-ent schemes. This aspect is discussed more in detail in Section 4, where the numerical examples show that such a condition is not necessary in the context of collocation. We note that, although in most of the literature the polynomial order of the basis functions and the orders of convergence are referred to as $p$, here we follow the notation in [32], where $p$ refers to the $p$-norm whereas $n$ refers to orders of consistency.

The HOLMES problem in (9) is solved by mean of duality methods, where the computation of the Lagrange multipliers is performed in an efficient and robust way using the Newton-Raphson scheme. The reader is referred to [32] for more details about the dual formulation and the computation of HOLMES first and second derivatives. A plot of HOLMES basis functions and their derivatives is given in Fig. 1 and Fig. 2, for the one and two-dimensional case respectively. It can be observed how the locality of the basis functions depends both on $\gamma$ and $p$ and, in particular, higher values of either $\gamma$ or $p$ lead to a more local character of the approximation. The figures also show the continuity of the basis functions, which are in general $C^{\infty}$-continuous if $p$ is even and $C^{p-1}$-continuous if $p$ is odd.

Strictly speaking, the support of HOLMES basis functions is the entire computational domain. 

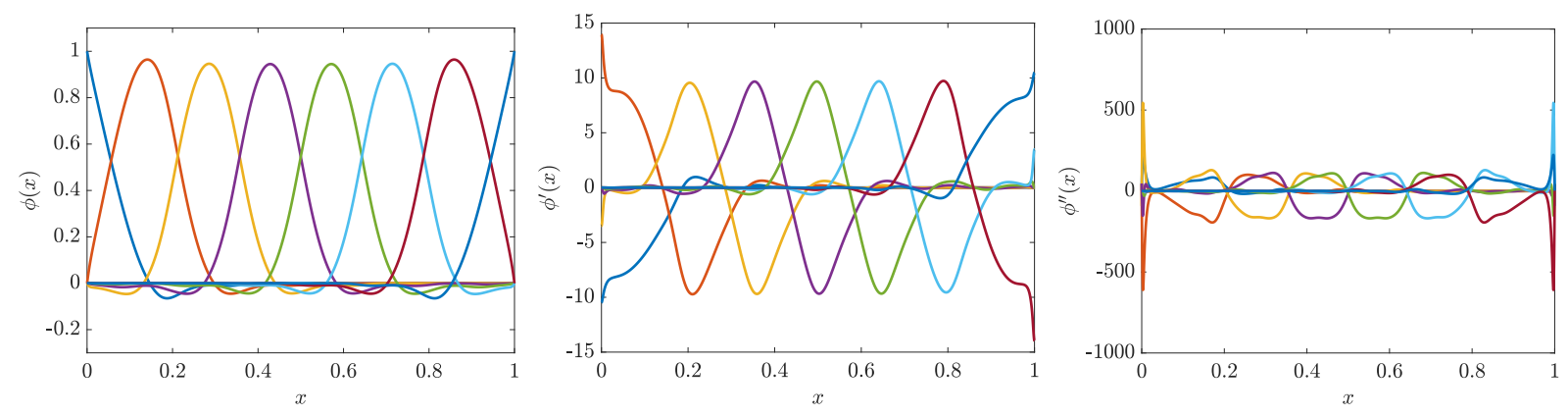

(a) $n=2, p=2, \gamma=1.521(\hat{R}=4)$
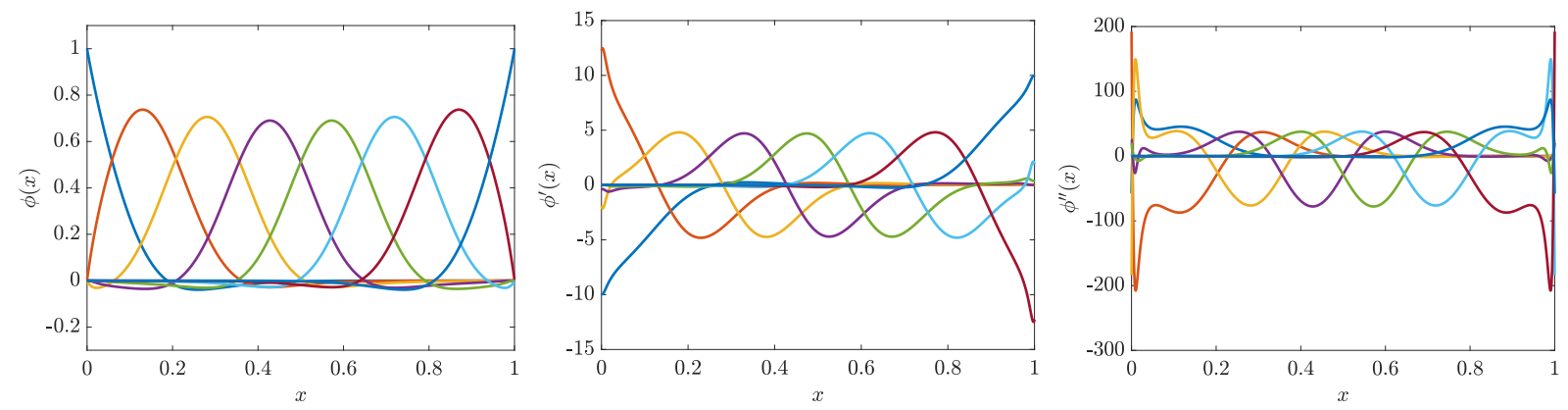

(b) $n=2, p=2, \gamma=0.676(\hat{R}=6)$
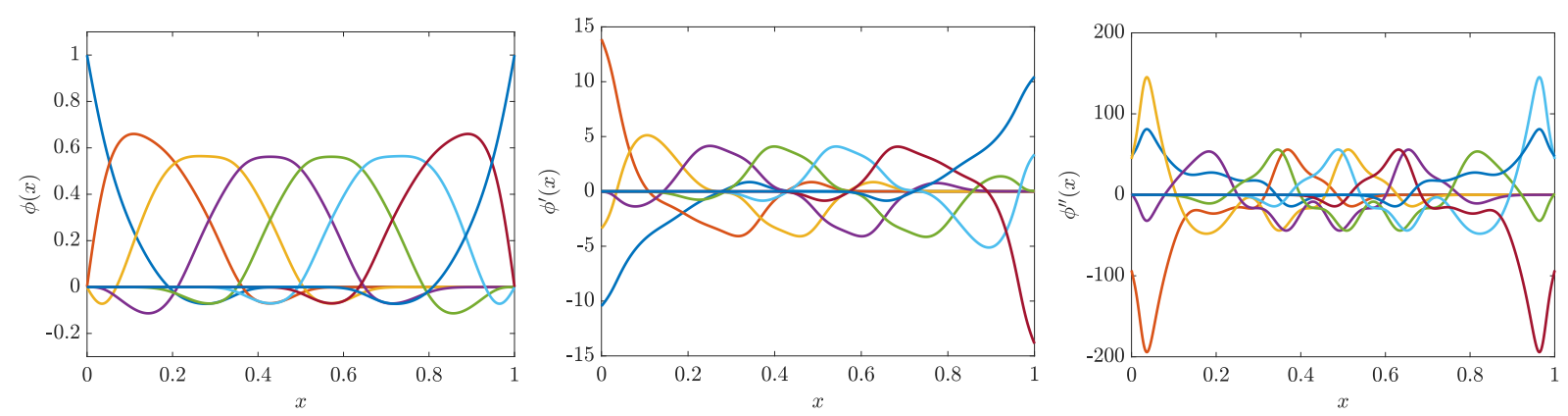

(c) $n=2, p=4, \gamma=0.095(\hat{R}=4)$
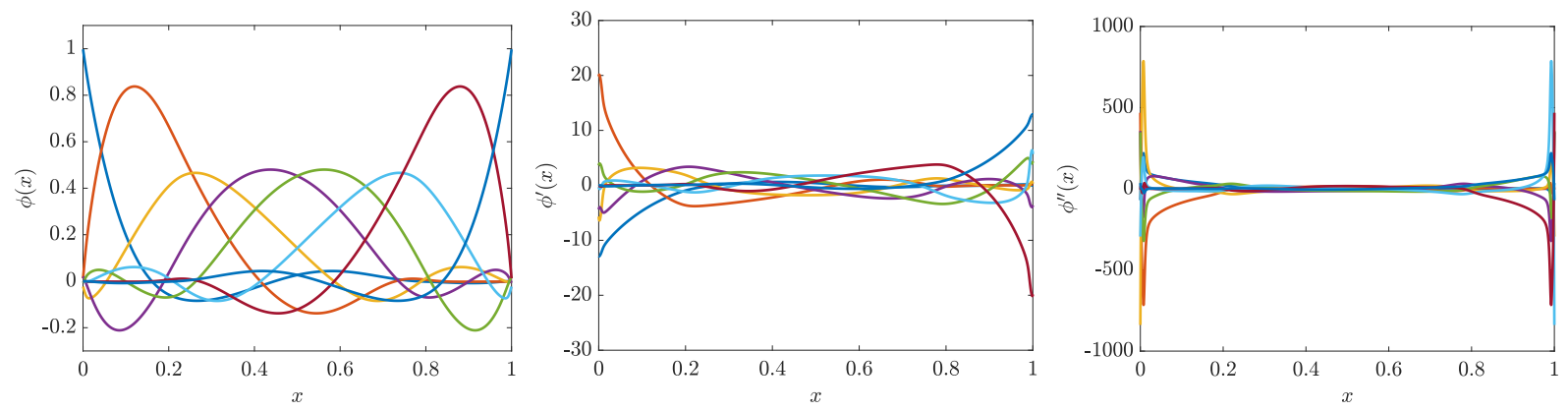

(d) $n=4, p=4, \gamma=0.006(\hat{R}=6)$

Figure 1: HOLMES basis functions in one dimension, computed for different orders $n$, different values of the locality norm $p$ and different estimated support sizes $\hat{R}$, for a truncation tolerance $\epsilon=10^{-11}$. 


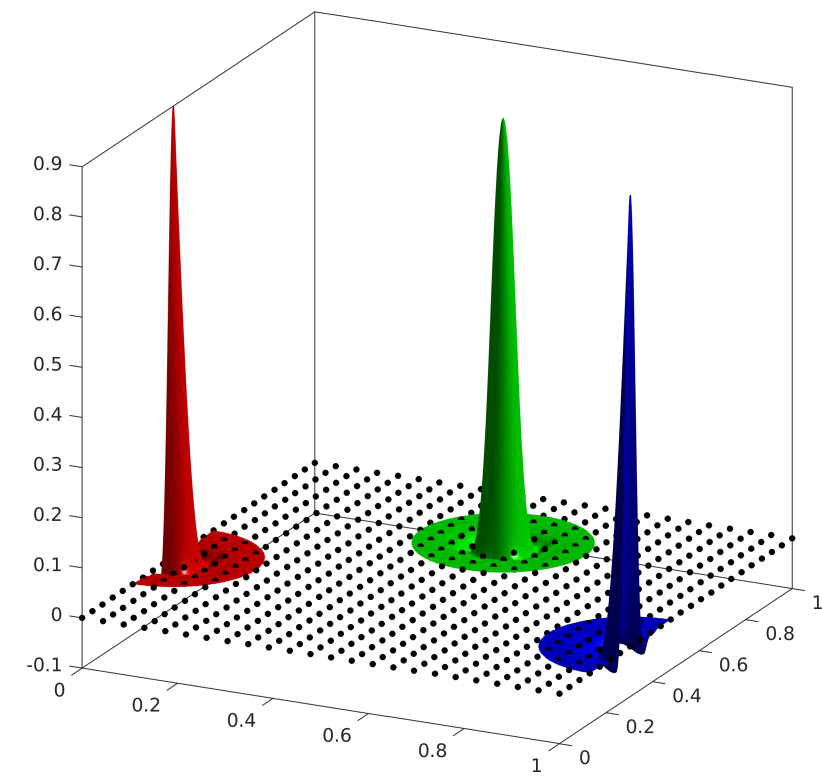

(a) $n=2, p=2, \gamma=1.521(\hat{R}=4)$

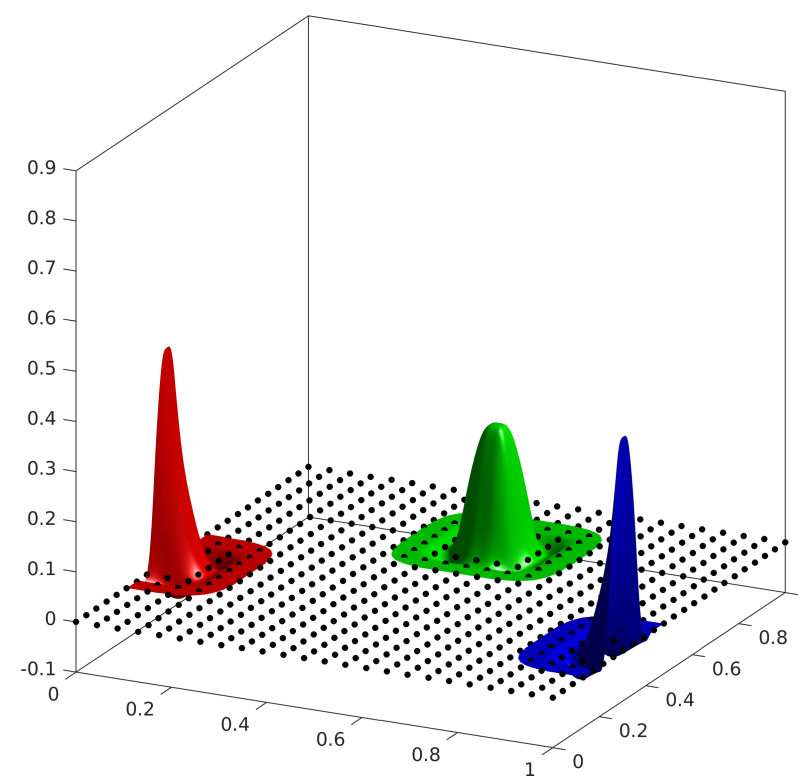

(c) $n=2, p=4, \gamma=0.095(\hat{R}=4)$

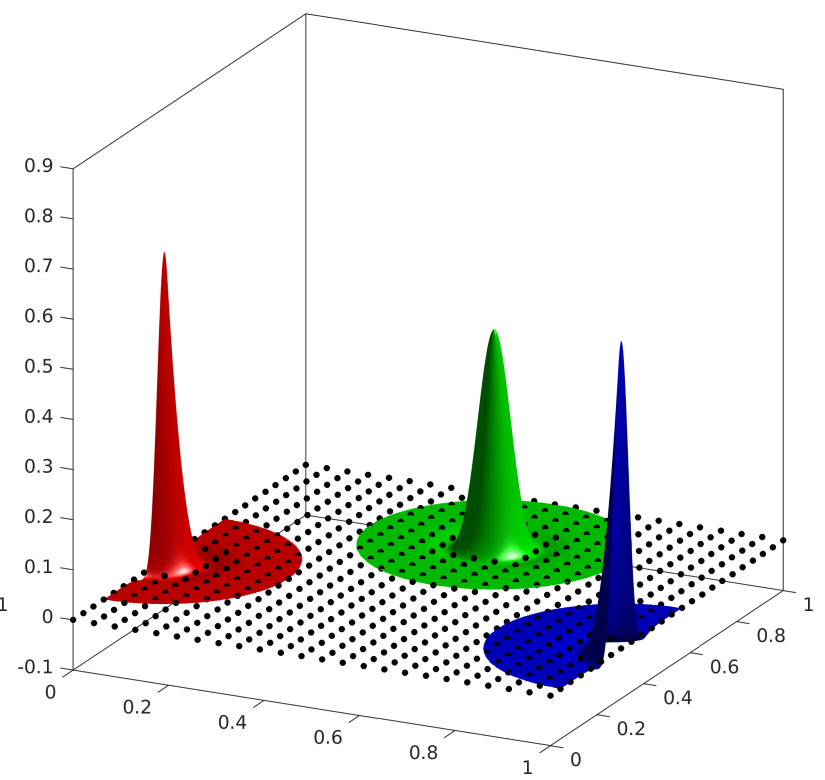

(b) $n=2, p=2, \gamma=0.676(\hat{R}=6)$

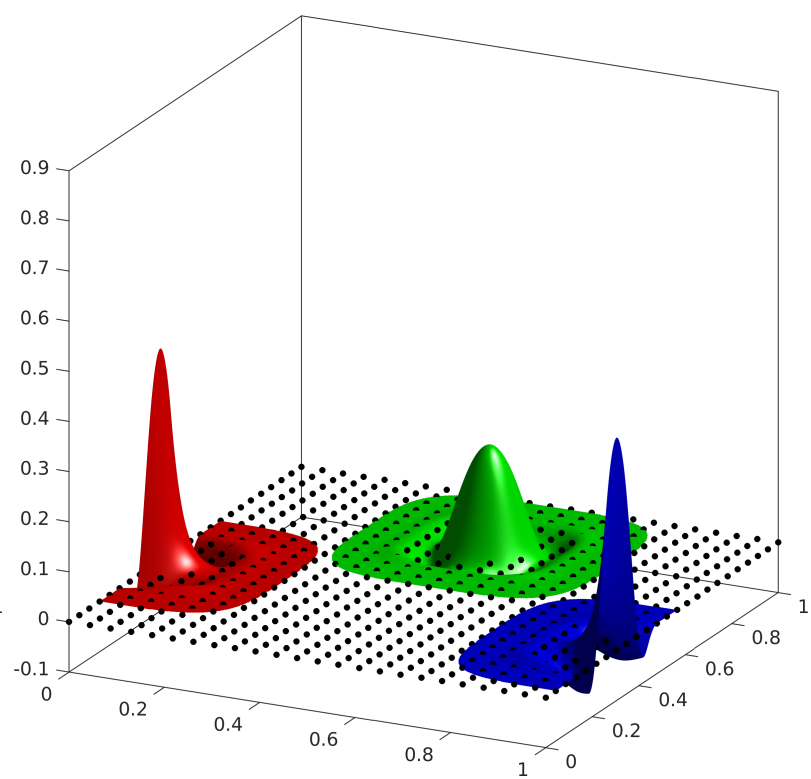

(d) $n=4, p=4, \gamma=0.006(\hat{R}=6)$

Figure 2: HOLMES basis functions in two dimensions, computed for different orders $n$, different values of the locality norm $p$ and different estimated support sizes $\hat{R}$, for a truncation tolerance $\epsilon=10^{-11}$. Note that the truncated support is circular only for $p=2$ and becomes square for $p \rightarrow \infty$. 
However, the locality term included in the formulation confers them with a fast exponential decay and, in the numerical practice, they can be truncated when their value is below a given threshold $\epsilon$. This is crucial to preserve the sparsity and the conditioning of the stiffness matrix. To implement the truncation, a common practice is to define an effective support of $\phi_{a}$ around $\mathbf{x}_{a}$ which, according to [32], can be approximated as

$$
r_{p}=h\left(-\frac{\log \epsilon+1}{\gamma}\right)^{\frac{1}{p}}
$$

Once this value is estimated, the basis functions are truncated when the $L^{p}$ distance from the evaluation point to a node is greater than $r_{p}$. Since the value of $r_{p}$ is proportional to the grid spacing $h$, a normalized support size $\hat{R}$ can be defined as

$$
\hat{R}=\frac{r_{p}}{h}=\left(-\frac{\log \epsilon+1}{\gamma}\right)^{\frac{1}{p}}
$$

and is used in this work to compare the different types of basis functions. Since their locality depends both on $\gamma$ and $p$, when $p$ changes different values of $\gamma$ are required to have the same support size, as it can be observed in Fig. 3. This is also noticeable in the basis functions plot of Fig. 1, where a radius $\hat{R}=4$ can be obtained either with $p=2$ and $\gamma=1.521$ (Fig. 1a) or with $p=4$ and $\gamma=0.095$ (Fig. 1c). Therefore, it is not straightforward to have a direct understanding of the basis functions character by looking only at the values of $\gamma$ and $p$. Here, for a given value of $p$, we fix the desired value of the support size $\hat{R}$ and the relative truncation tolerance $\epsilon$ and, then, compute the required $\gamma$ as

$$
\gamma=-\frac{\log \epsilon+1}{\hat{R}^{p}}
$$

The obtained value of $\gamma$ is then employed to compute the basis functions.

\section{Numerical examples}

The performance of HOLMES collocation is studied in this section through a selection of numerical examples. Given the large amount of parameters involved in the computation of HOLMES basis functions, a one-dimensional application is considered first, in order to have a preliminary understanding of their effect on the convergence and accuracy of the method. Then, benchmark applications for acoustic Helmholtz problems and for linear elasticity are considered. Finally, the application of HOLMES collocation to domains defined by a NURBS curve is discussed more in detail. 


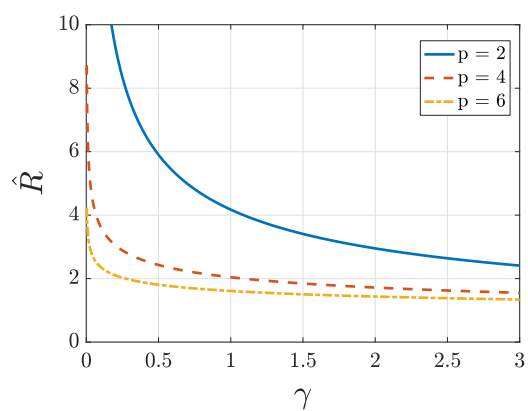

(a) $\epsilon=10^{-8}$

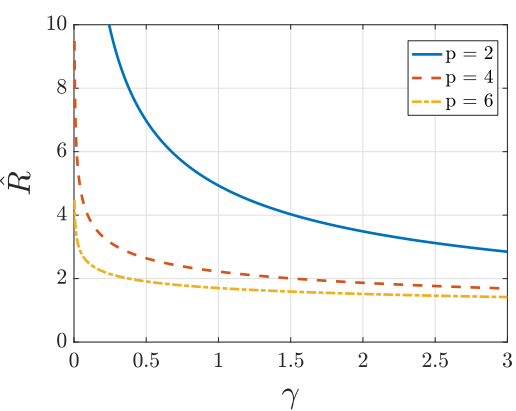

(b) $\epsilon=10^{-11}$

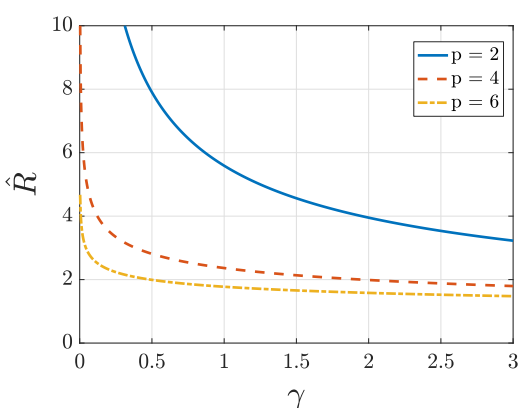

(c) $\epsilon=10^{-14}$

Figure 3: Dependence of the normalized support size of HOLMES basis functions with respect to $\gamma$ and $p$, for different values of the truncation tolerance $\epsilon$.

In all the examples, relative discrete errors are considered for the convergence studies, which for a given vector field $\mathbf{v}(\mathbf{x})$ and its numerical approximation $\mathbf{v}^{h}(\mathbf{x})$, are defined as

$$
E_{r}\left(\mathbf{v}, \mathbf{v}^{h}\right)=\sqrt{\frac{\sum_{a=1}^{m}\left(\mathbf{v}_{a}-\mathbf{v}_{a}^{h}\right)\left(\mathbf{v}_{a}-\mathbf{v}_{a}^{h}\right)^{T}}{\sum_{a=1}^{m} \mathbf{v}_{a} \mathbf{v}_{a}^{T}}},
$$

where $\mathbf{v}_{a}$ and $\mathbf{v}_{a}^{h}$ are respectively the values of $\mathbf{v}$ and $\mathbf{v}^{h}$ at the node $\mathbf{x}_{a}$.

As far as theory is concerned, an abstract mathematical framework for the numerical analysis of collocation methods has not been developed yet. For meshfree collocation, some preliminary results can be found in $[21,22,42,43]$. In the case of IGA, a thorough theoretical analysis that includes proofs of stability, convergence and error estimates is available for the one-dimensional case in [2]. For higher dimensions, convergence results and error estimates are available only in numerical studies $[2,3]$. Based on these references, a-priori estimates for the convergence rate are used in this work to validate the numerical results. In particular, for a given order of consistency $n$, the approximate solution is expected to converge with a rate of $n$ for the error in both the $L^{2}$ norm and the $H^{1}$ semi-norm if $n$ is even, and with a rate of $n-1$ for both norms if $n$ is odd.

\subsection{Preliminary study in one dimension}

A one-dimensional problem, stated by the following Helmholtz equation is considered:

$$
\Delta u(x)+u(x)=b(x), \quad x \in[-1 ; 1],
$$

where $b(x)$ is a source term compatible with the following solution

$$
u(x)=\sin (3 x) \exp (x)+\tan ^{-1}(x)+\cosh (x) .
$$


This PDE is solved together with suitable boundary conditions to recover $u(x)$. Two sets of boundary-value problems are considered, with either essential or natural boundary conditions, and the corresponding convergence curves of the relative discrete $L^{2}$ error, $E_{r}\left(u, u^{h}\right)$, as a function of the number of nodes $m$ are plotted in Fig. 4 and Fig. 5 respectively.

The convergence study considers HOLMES approximants of different orders $(n=2,4,6)$ and different values of the locality parameters $\gamma$ and $p$. As discussed in Section 3 , for each $p$ the values of $\gamma$ are chosen such that values of the normalized support size from $\hat{R}=n$ to $\hat{R}=n+4$ are obtained, for a fixed truncation tolerance of $\epsilon=10^{-11}$. It has to be remarked that, for HOLMES of a given order $n$, at least $n+1$ nodes are necessary in the computation of the basis functions for problem (9) to be well-posed. Therefore, the nodes on the boundary need $\hat{R} \geq n$ to verify this condition.

Analyzing the results relative to essential boundary conditions in Fig. 4, it can be observed how the curves converge in all the cases, but the results become slightly worse for higher values of the locality norm $p$. This situation becomes more evident for the case of natural boundary conditions in Fig. 5, where for $p=2$ the expected convergence is obtained, whereas for higher $p$ the curves still converge but with a much more irregular behavior. The only exception for $\hat{R}=n$, where a few curves do not converge when $n=4$ and $n=6$.

As mentioned earlier, in the context of Galerkin formulations the condition $p>n$ is sufficient for the convergence of the method since it ensures that the basis functions have a proper exponential decay [32]. Here, converging solutions are obtained for $p \leq n$. Furthermore, the numerical results presented in this section suggest that HOLMES collocation has a better convergence behavior for $p=2$. This result can be explained by observing the basis functions derivatives plotted in Fig. 1, which are more irregular for higher values of $p$ particularly close to the boundary.

A possible explanation for the non-converging curves in the case of $\hat{R}=n$ can be that the corresponding values of $\gamma$ do not give enough overlap to the basis functions and, therefore, for each collocation node the values of the basis functions of the furthest nodes and their relative second derivatives are too small. Indeed, it is worth noting that the numerical truncation, defined through Eq. (12), is only an approximation of the decay of the basis functions and, in addition, the behavior of the second derivatives is slightly different, particularly for higher values of $p$. This can also explain why for $n=6$ the convergence curve stagnates around $10^{-5}$ for $p=2$ but does 


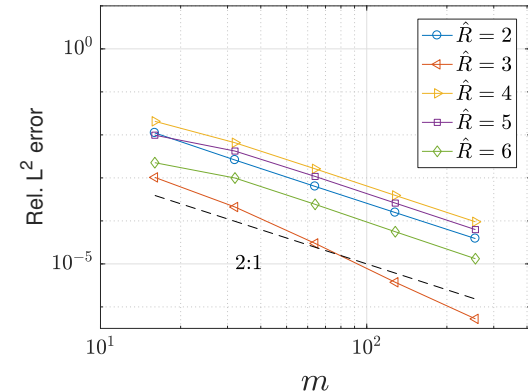

(a) $n=2, p=2$

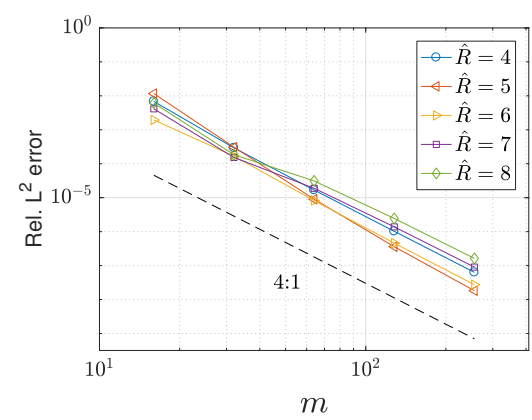

(d) $n=4, p=2$

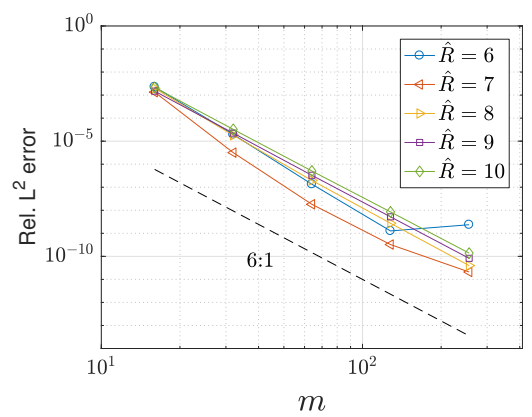

(g) $n=6, p=2$

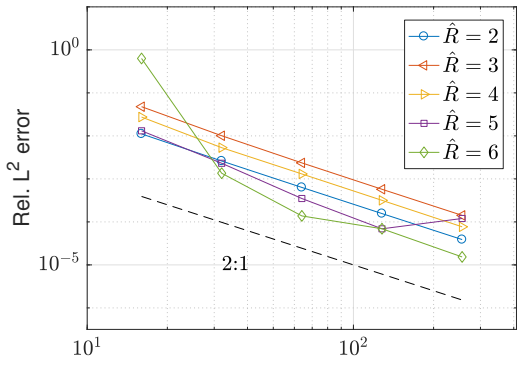

m

(b) $n=2, p=4$

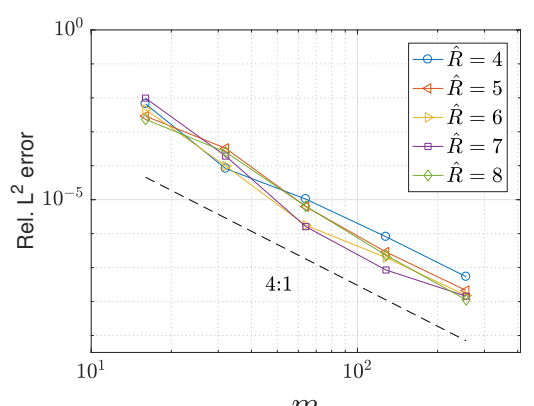

(e) $n=4, p=4$

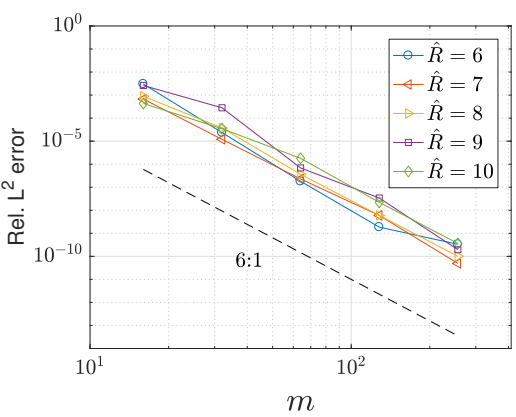

(h) $n=6, p=4$

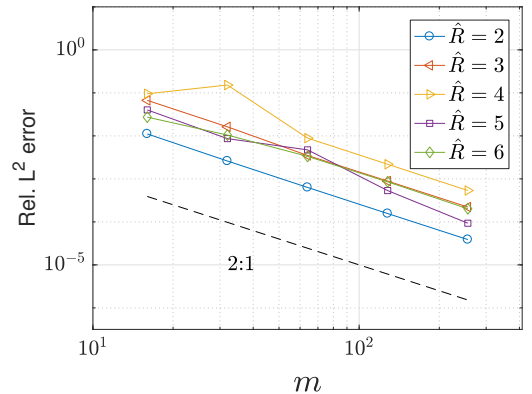

(c) $n=2, p=6$

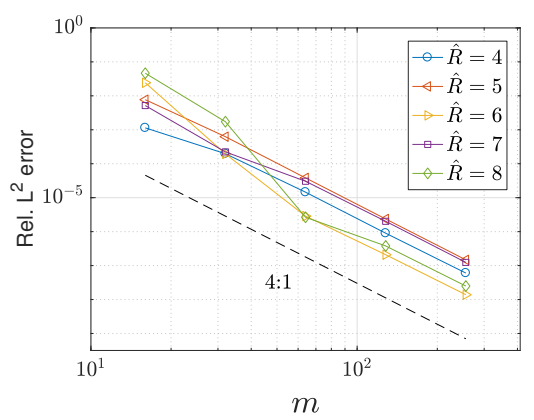

(f) $n=4, p=6$

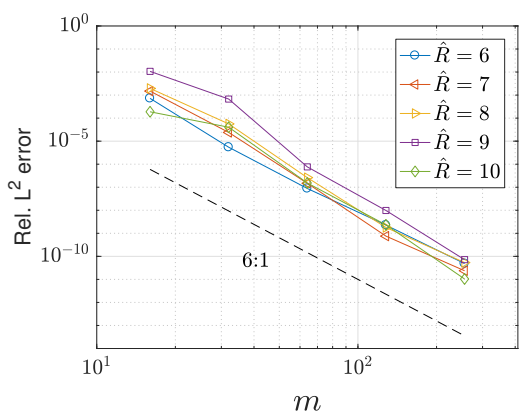

(i) $n=6, p=6$

Figure 4: Convergence of the relative $L^{2}$ error for a 1D Helmholtz problem with essential boundary conditions solved for different values of $n$ and $p$ and a truncation tolerance $\epsilon=10^{-11}$. 


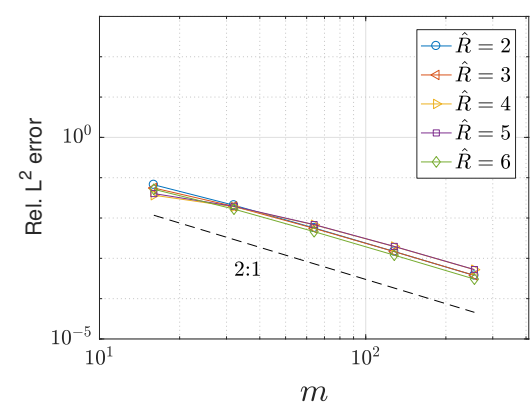

(a) $n=2, p=2$

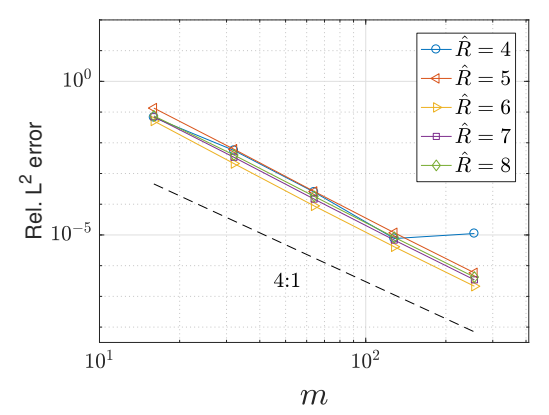

(d) $n=4, p=2$

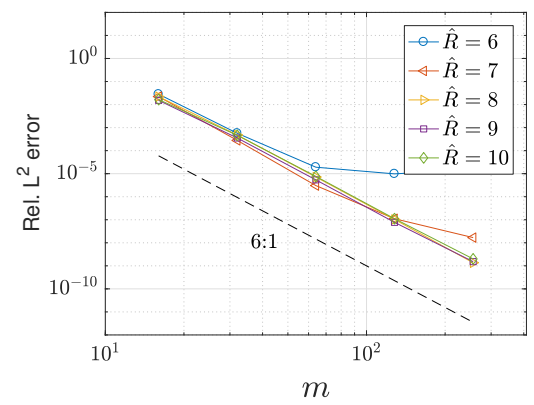

(g) $n=6, p=2$

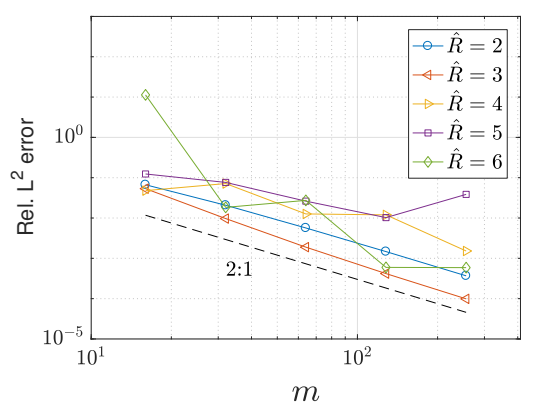

(b) $n=2, p=4$

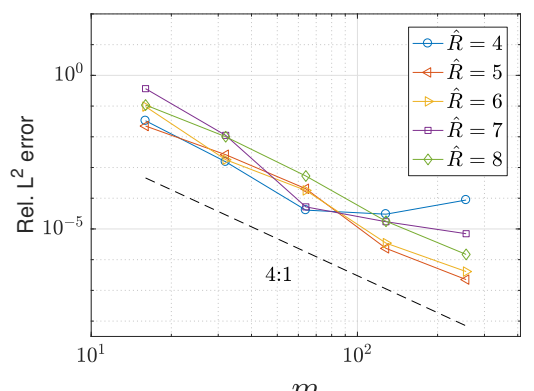

(e) $n=4, p=4$

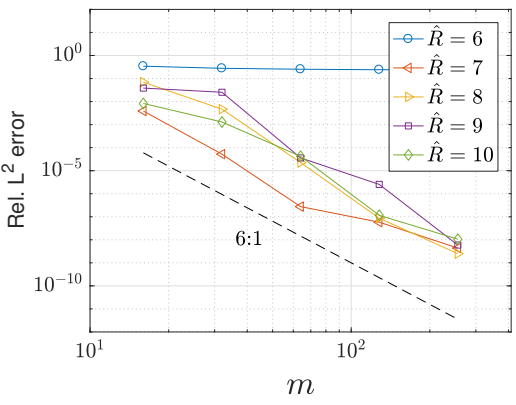

(h) $n=6, p=4$

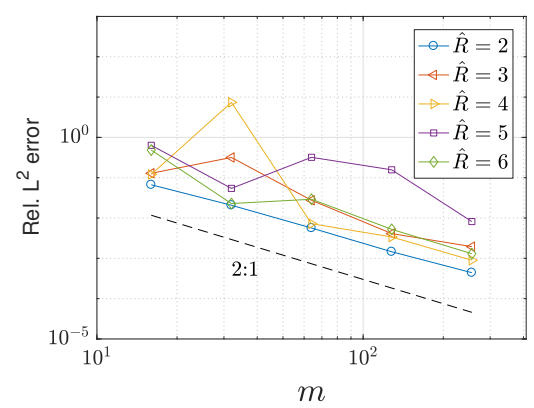

(c) $n=2, p=6$

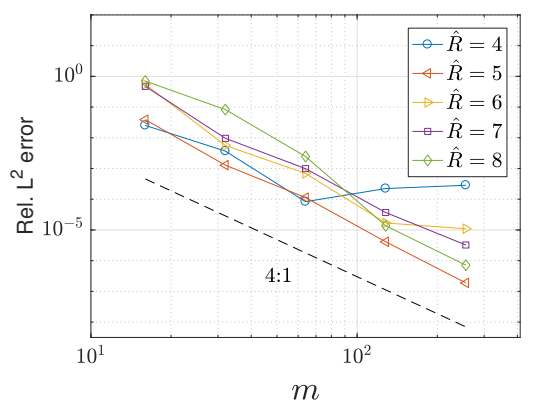

(f) $n=4, p=6$

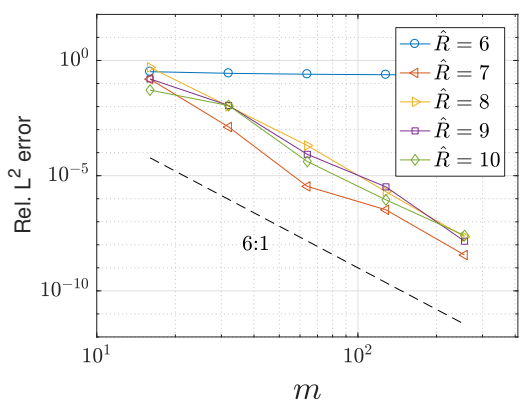

(i) $n=6, p=6$

Figure 5: Convergence of the relative $L^{2}$ error for a 1D Helmholtz problem with natural boundary conditions solved for different values of $n$ and $p$ and a truncation tolerance $\epsilon=10^{-11}$. 


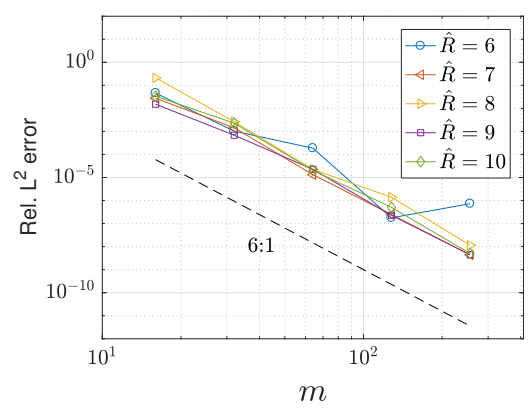

(a) $\epsilon=10^{-8}$

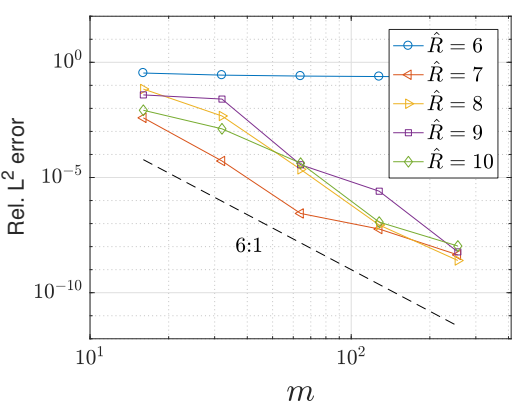

(b) $\epsilon=10^{-11}$

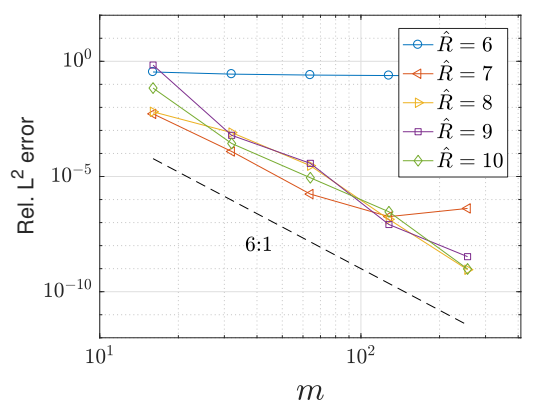

(c) $\epsilon=10^{-14}$

Figure 6: Convergence of the relative $L^{2}$ error for a 1D Helmholtz problem with natural boundary conditions, solved for $n=6, p=4$ and different values of the truncation tolerance $\epsilon$.

not converge for higher values of $p$. This consideration is confirmed by the convergence curves in Fig. 6, where the case of $n=6$ and $p=4$ from Fig. $5 \mathrm{~h}$ is analyzed for different values of the truncation tolerance $\epsilon$. It can be observed in Fig. 6a how a higher tolerance, which corresponds to lower values of $\gamma$, is indeed changing the behavior of the non-converging curve for $\hat{R}=6$. However, on using higher values of $\epsilon$, the truncation error may affect the bottom part of the convergence curves with an effect which is difficult to estimate. Taking this into account, the value of $\epsilon=10^{-11}$ is still used in the following numerical examples.

In all the converging curves, the expected convergence rate of $n$ is obtained. Similar results, in terms of a higher irregularity with higher values of $p$, are also obtained for odd values $(n=3$ and $n=5$ ), which also exhibit the expected convergence rates of $n-1$. A summarizing convergence plot is presented in Fig. 7 with HOLMES of order $n$ from 2 to 6 and the two types of boundary conditions. For this plot, a value of $\hat{R}=n+2$ is chosen for all the curves. It is worth noting that, according to Figures 4 and 5, the performance of HOLMES approximants is in general strongly dependent on the locality parameter $\gamma$, with lower values of $\gamma$ corresponding to a higher support size $\hat{R}$. For each specific case, an optimal value of $\gamma$ exists, below which the accuracy is anyway decreasing. Indeed, based on the curves in the figures, there not seem to be a single optimal value of $\hat{R}$ for all the configurations. Therefore, $\hat{R}=n+2$ is chosen.

In Fig. 7 it can be observed how the odd orders have a very similar behavior to the corresponding $n-1$ even orders but, remarkably, the computational times to compute the basis functions are higher due to the higher number of constraints to be imposed in problem (9). 


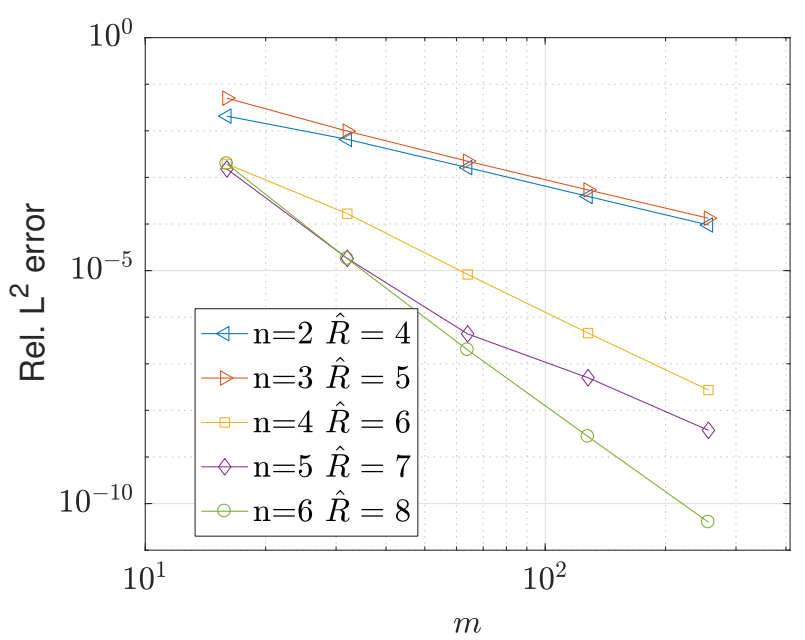

(a) Essential BCs

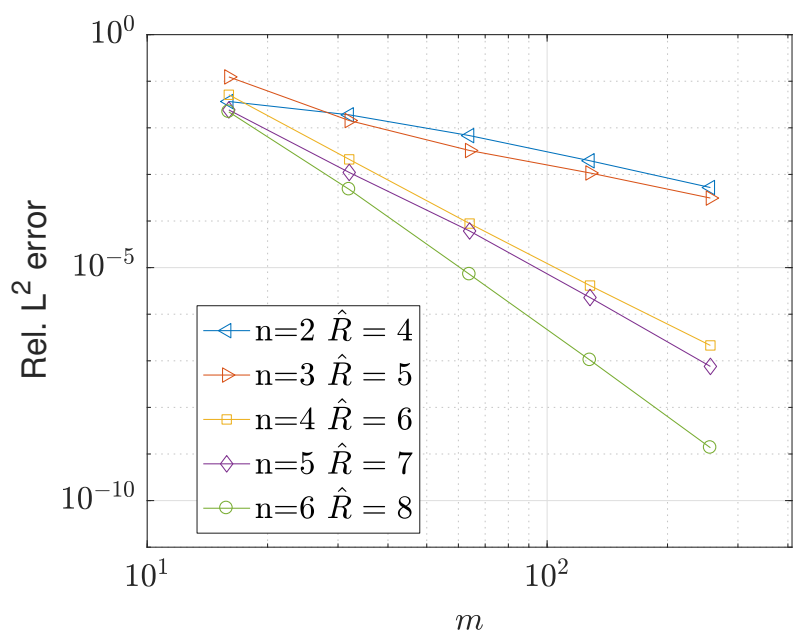

(b) Natural BCs

Figure 7: Convergence of the relative $L^{2}$ error for a 1D Helmholtz problem, with different types of boundary conditions, solved for different values of $n$ and $p=2$.

\subsection{Acoustic Helmholtz problems}

This section considers the propagation of acoustic waves, described by the following Helmholtz problem:

$$
\begin{cases}\Delta p+k^{2} p=0 & \text { in } \Omega \\ \frac{\mathrm{j}}{\rho_{0} \omega} \nabla p \cdot \boldsymbol{n}=\bar{v}_{n} & \text { on } \partial \Omega,\end{cases}
$$

where $p=p(\mathbf{x})$ is the acoustic pressure field, $\rho_{0}$ is the fluid-medium density, $\mathbf{j}$ is the imaginary unit and $k$ is the wavenumber, which is related to the angular frequency $\omega$ and the wavelength $\lambda$ by the speed of sound $c$, through the following equations:

$$
k=\frac{\omega}{c}=\frac{2 \pi}{\lambda} .
$$

The problem is first solved in two dimensions in a circular domain, where it describes the acoustic field generated within a cylinder with infinite vibrating walls. The analytical expression of the pressure is given by

$$
p(r)=-\bar{v}_{n} \frac{J_{0}(k r)}{\frac{j}{\rho_{0} \omega} k J_{1}(k R)},
$$

where $J_{0}$ and $J_{1}$ are the Bessel functions of the first kind of order 0 and 1 respectively [53]. The following parameters are considered: $R=1 \mathrm{~m}, \rho_{0}=1.21 \mathrm{~kg} / \mathrm{m}^{3}, c=343 \mathrm{~m} / \mathrm{s}, \bar{v}_{n}=1 \mathrm{~m} / \mathrm{s}$ and the problem is solved for a wavelength $\lambda=2.5 \mathrm{~m}$, corresponding to $\omega=862.05 \mathrm{rad} / \mathrm{s}$. The 


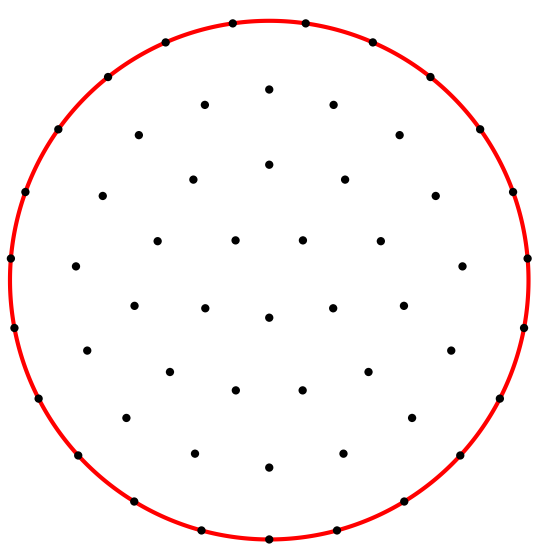

(a)

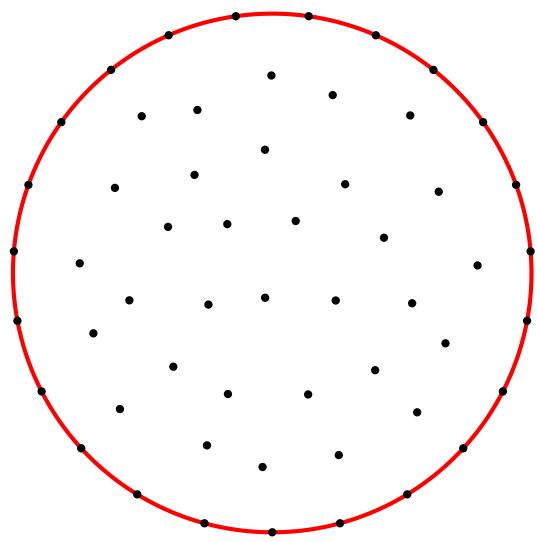

(c)

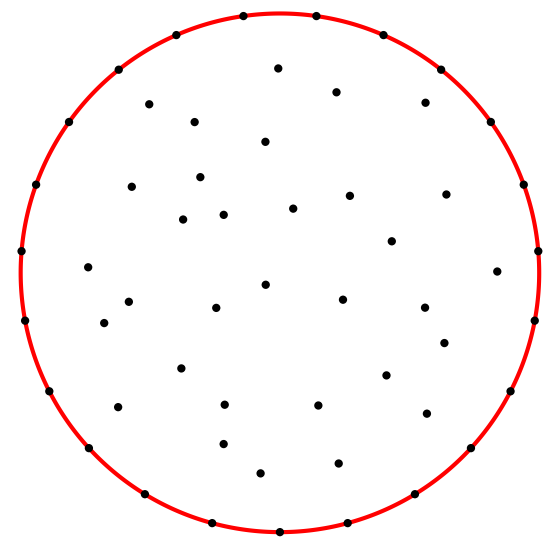

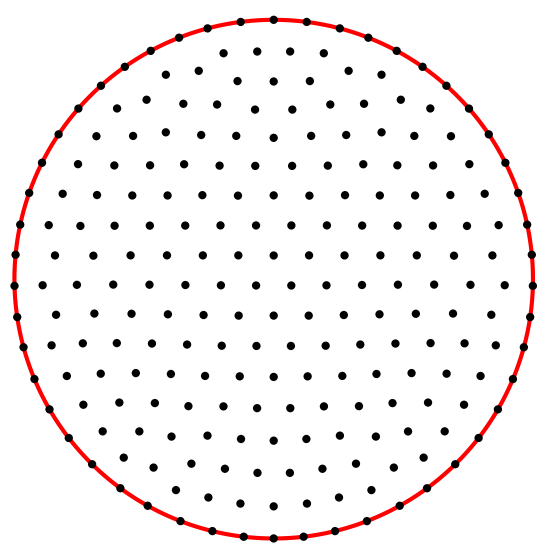

(b)

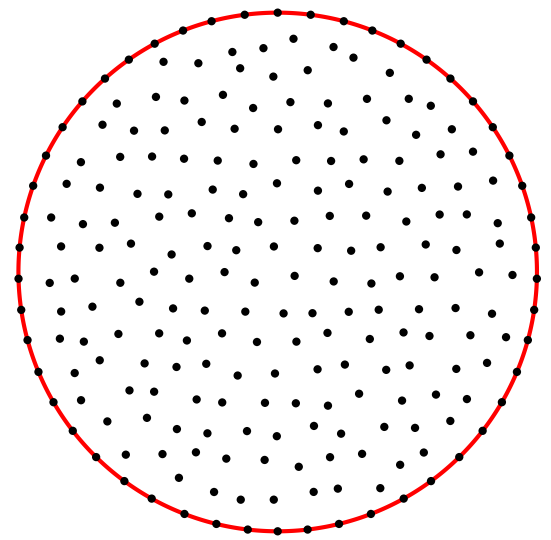

(d)

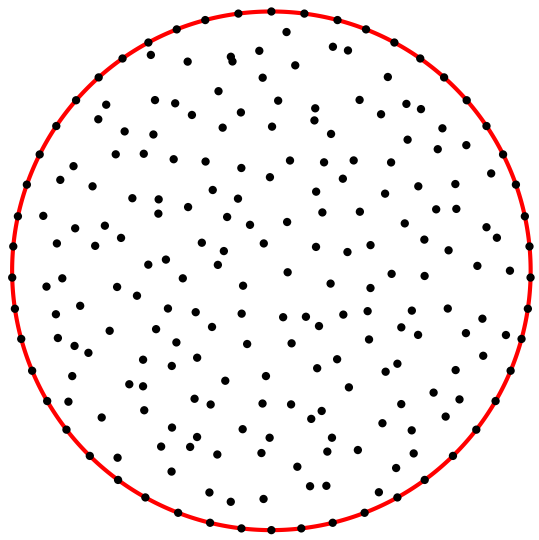

(e)

Figure 8: Nodal discretizations for the Helmholtz problem on a circular domain. The first two grids (a) and (b), with 55 and 229 nodes respectively, are generated with the Matlab mesher distmesh. To obtain irregular distributions, the internal nodes are moved by adding a random displacement perturbation whose components are in the range of $[-0.2 h 0.2 h]$ in (c) and (d) and $[-0.4 h 0.4 h]$ in (e) and (f). 


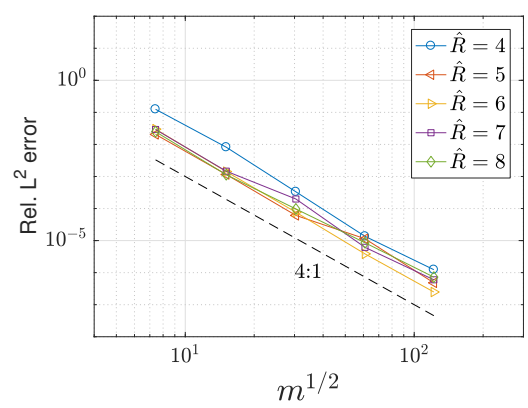

(a) $n=4, p=2$

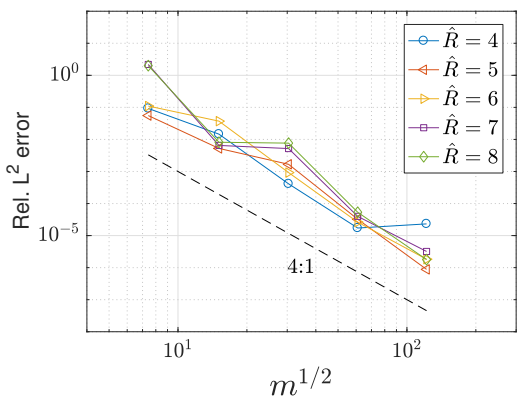

(b) $n=4, p=4$

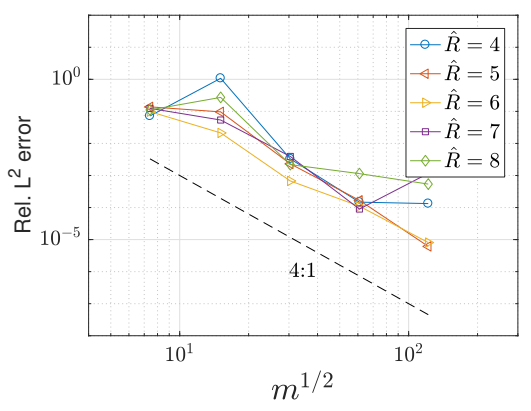

(c) $n=4, p=6$

Figure 9: Convergence of the relative $L^{2}$ and $H^{1}$ error for the 2D acoustic Helmholtz problem solved for $n=4$ and different values of and $p$.

computational grid on the circular domain is generated using the MATLAB finite element mesh generator distmesh, which provides very regular nodal distributions [54], as outlined in Fig. 8 a-b.

Convergence curves of the relative discrete $L^{2}$ error, $E_{r}\left(u, u^{h}\right)$, in function of the square root of the number of nodes $m^{1 / 2}$ are plotted in Fig. 9, for HOLMES approximants of order $n=4$ and different values of $p$ and $\hat{R}$. This two-dimensional application confirms that a more regular convergence behavior is obtained for $p=2$ and similar results, not included for the sake of brevity, are found for HOLMES of different orders. In Fig. 10a, orders from 2 to 6 with $p=2$ are considered and are shown to test the expected convergence rates for the $L^{2}$ error. Unlike the one-dimensional case, HOLMES of odd order show better accuracy than the corresponding $n-1$ even orders, but the asymptotic rates still verify the theoretical prediction of a slope given by $n-1$. Fig. 10b considers also the relative error in the discrete $H^{1}$ semi-norm, which is defined according to Eq. (14) as $E_{r}\left(\nabla u, \nabla u^{h}\right)$. The expected convergence rates, equal to those of the $L^{2}$ error, are observed.

To study the performance of HOLMES collocation on irregular nodal distributions, the grids of the convergence study are perturbed by adding a random displacement to the internal nodes, as shown in Fig. 8 c-f. Two cases are considered with an added displacement whose components are in the range of $[-0.2 h 0.2 h]$ and $[-0.4 h 0.4 h]$ respectively. The corresponding convergence curves for the relative discrete $L^{2}$ and $H^{1}$ errors are reported in Fig. 10 c-f. Although a slight decrease of accuracy can be observed, the curves converge with the expected rates in both cases, which confirms the validity of the collocation approach when using irregular nodal distributions. Such configurations can arise for instance in dynamical large deformation problems.

The Helmholtz problem stated by Eq. (17) is studied also in three dimensions to compute the 


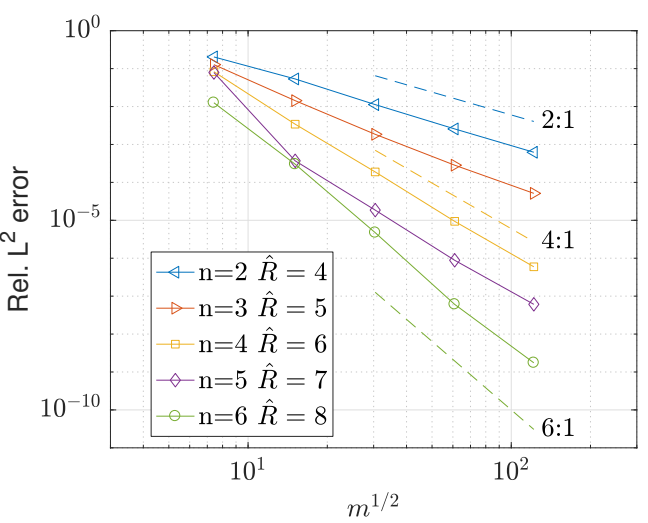

(a)

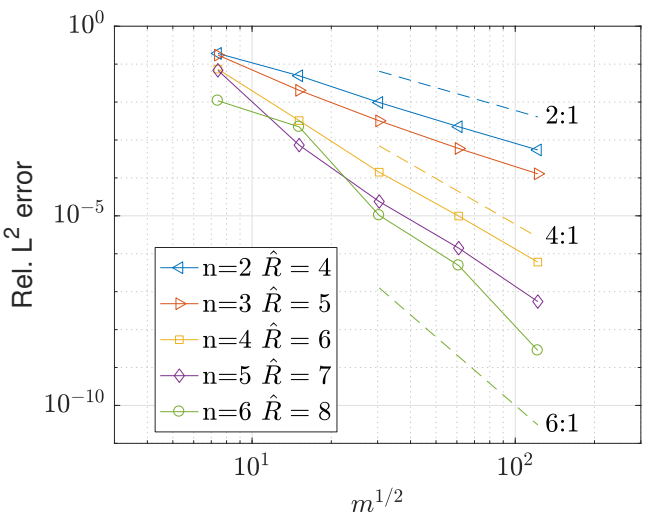

(c)

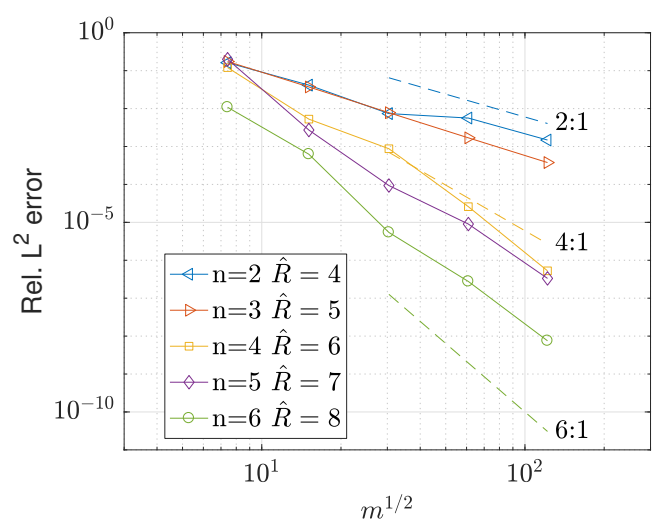

(e)

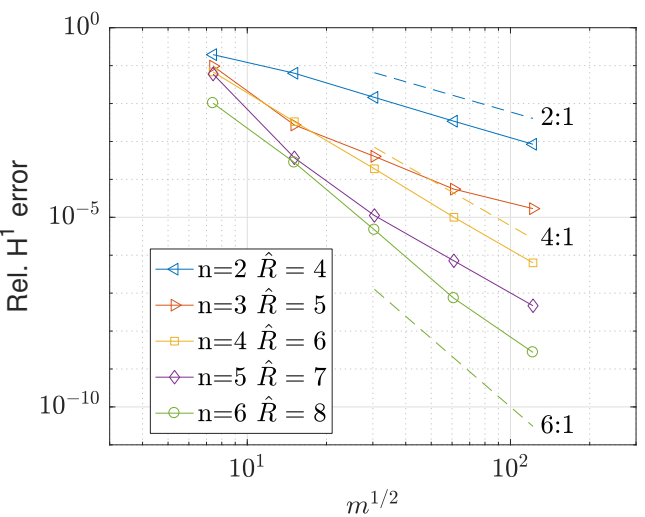

(b)

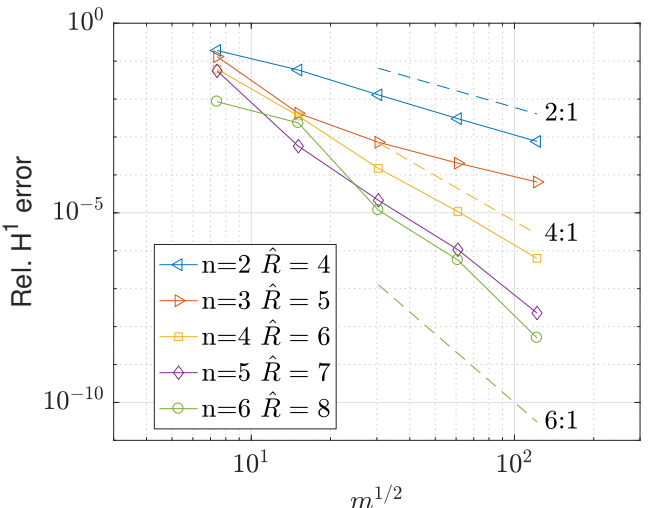

(d)

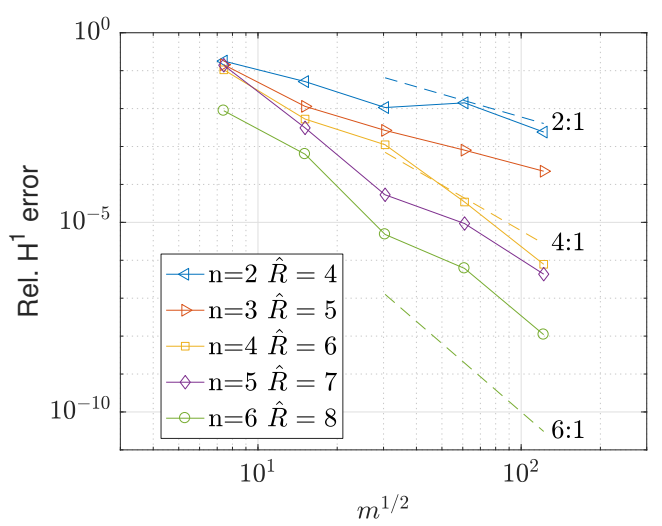

(f)

Figure 10: Convergence of the relative $L^{2}$ and $H^{1}$ error for the 2D acoustic Helmholtz problem solved for different values of $n$ and $p=2$. Regular nodal distributions are considered in (a) and (b), while a perturbation of $0.2 h$ is considered in (c) and (d) and a perturbation of $0.4 h$ is considered in (e) and (f). 


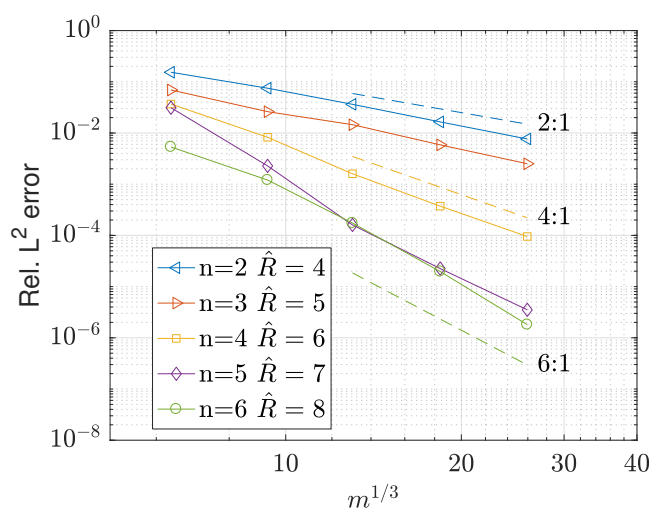

(a)

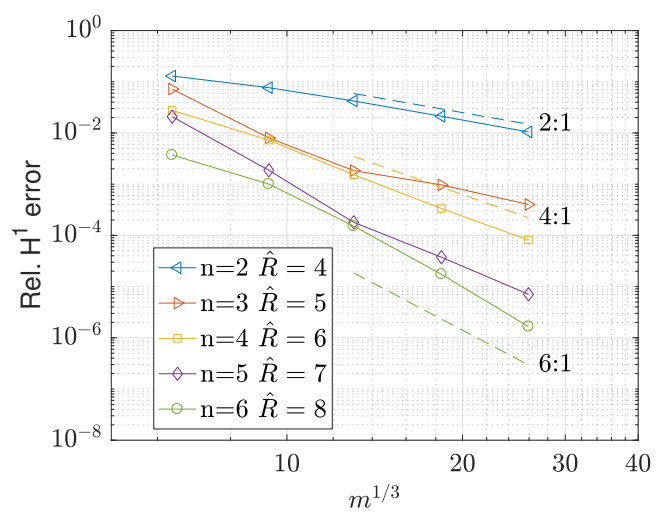

(b)

Figure 11: Convergence of the relative $L^{2}$ and $H^{1}$ error for the 3D acoustic Helmholtz problem solved for different values of $n$ and $p=2$.

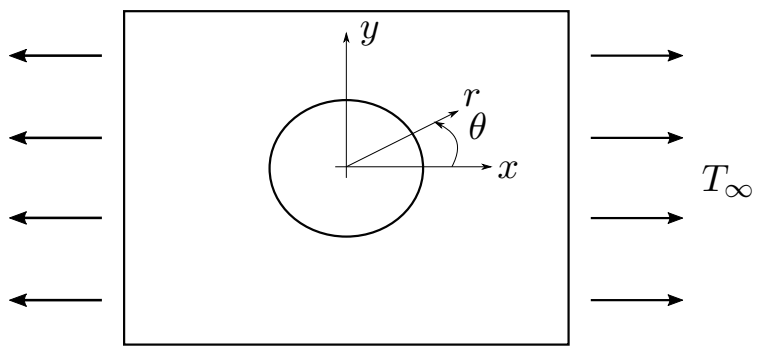

Figure 12: Elastic plate with a circular hole under tension.

acoustic field inside a sphere with vibrating walls of radius $R=1 \mathrm{~m}$. The same acoustic parameters and wavelength of the $2 \mathrm{D}$ case are considered. In three dimensions, the analytical solution of the problem is given by

$$
p(r)=-\mathrm{j} \bar{v}_{n} \rho_{0} \omega \frac{e^{-\mathrm{j} k(r-1)}\left(e^{2 \mathrm{j} k r}-1\right)}{\left[e^{2 \mathrm{j} k}(\mathrm{j} k-1)+\mathrm{j} k+1\right] r} .
$$

The convergence results are reported in Fig. 11 for HOLMES of orders $n$ from 2 to 6 and a locality norm $p=2$. A similar convergence behavior to the $2 \mathrm{D}$ case is obtained and the expected convergence rates are verified for both the $L^{2}$ and the $H^{1}$ error.

\subsection{Linear elasticity}

\subsubsection{Infinite elastic plate with hole}

This section considers the well known benchmark problem of an infinite plate with a circular hole, subject to an uni-axial traction $T_{\infty}$ on the remote boundary, as outlined in Fig. 12. The 
analytical solution for the displacement field is given in polar coordinates as [55]

$$
\begin{aligned}
& u(r, \theta)=T_{\infty} \frac{R}{8 G}\left\{\frac{r}{R}(\kappa+1) \cos \theta+\frac{2 r}{R}[(1+\kappa) \cos \theta+\cos 3 \theta]-\frac{2 r^{3}}{R^{3}} \cos 3 \theta\right\}, \\
& v(r, \theta)=T_{\infty} \frac{R}{8 G}\left\{\frac{r}{R}(\kappa-3) \sin \theta+\frac{2 r}{R}[(1-\kappa) \sin \theta+\sin 3 \theta]-\frac{2 r^{3}}{R^{3}} \sin 3 \theta\right\},
\end{aligned}
$$

being $G$ the shear modulus and $\kappa$ the Kolosov constant. In particular, a state of plane stress is assumed, such that it takes the value

$$
\kappa=\frac{3-\nu}{1+\nu} .
$$

The corresponding stress distribution is given by

$$
\begin{aligned}
\sigma_{x}(r, \theta) & =T_{\infty}\left[1-\frac{R^{2}}{r^{2}}\left(\frac{3}{2} \cos 2 \theta+\cos 4 \theta\right)+\frac{3 R^{4}}{2 r^{4}} \cos 4 \theta\right], \\
\sigma_{y}(r, \theta) & =T_{\infty}\left[-\frac{R^{2}}{r^{2}}\left(\frac{1}{2} \cos 2 \theta-\cos 4 \theta\right)-\frac{3 R^{4}}{2 r^{4}} \cos 4 \theta\right], \\
\tau_{x y}(r, \theta) & =T_{\infty}\left[-\frac{R^{2}}{r^{2}}\left(\frac{1}{2} \sin 2 \theta+\sin 4 \theta\right)+\frac{3 R^{4}}{2 r^{4}} \sin 4 \theta\right] .
\end{aligned}
$$

The problem is solved imposing essential boundary conditions, for a value of $T_{\infty}=1$ and taking as material parameters $E=1$ and $\nu=0.3$. A computational domain $[-4,4] \times[-4,4]$, with an interior circle of radius $R=1$ is considered and, because of symmetry, only a quarter of the plate is studied. Two types of discretizations are used: regular computational grids created with the distmesh generator and irregular ones obtained by adding a nodal perturbation whose components are in the range of $[-0.2 h 0.2 h]$, as outlined in Fig. 13.

The convergence of the relative $L^{2}$ error of the displacement and stress fields in function of the square root of the number of nodes $m^{1 / 2}$ are reported in Fig. 14. It can be observed how the expected convergence rates are obtained for both the displacement and the stress fields. For the latter, we note that the stress error in the $L^{2}$ norm is expected to converge as a displacement error in the $H^{1}$ semi-norm. Remarkably, also for this problem, the irregular nodal distribution provides only a small accuracy shift of some curves, without altering the correct convergence rates.

\subsubsection{L-shaped plate with stress singularity}

In order to better investigate the performance of HOLMES collocation also for problems with singularities, this section considers an elastic body with a re-entrant corner subjected to tractions on remote boundaries, as shown in Fig. 15. The analytical solution in the vicinity of the singular 


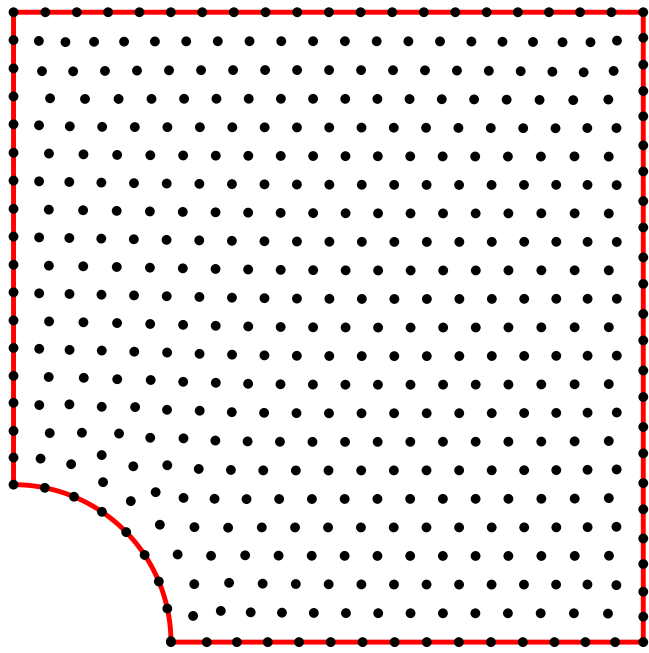

(a)

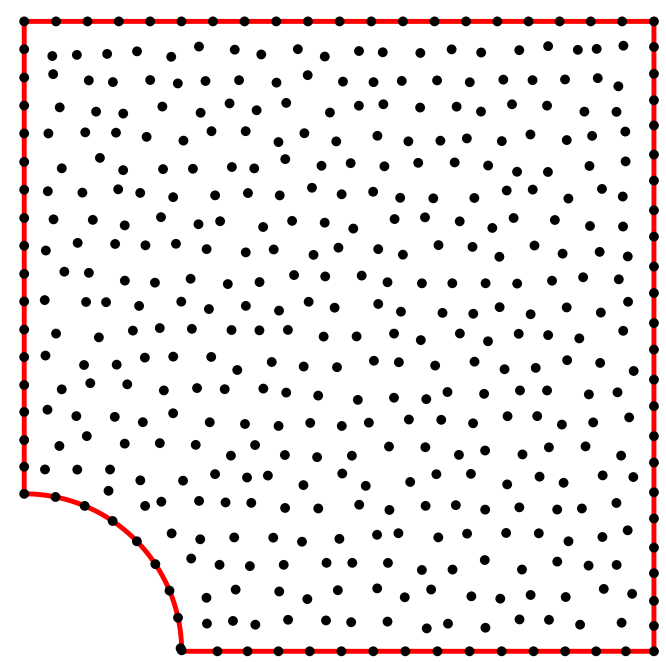

(c)

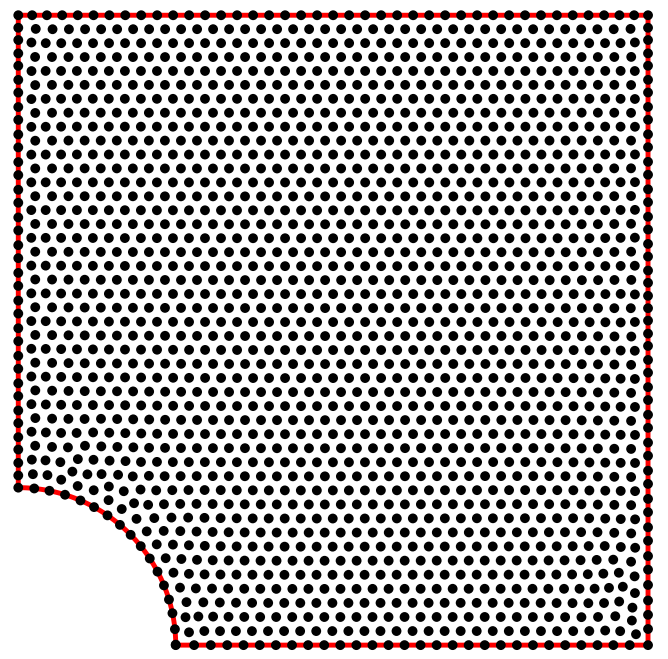

(b)

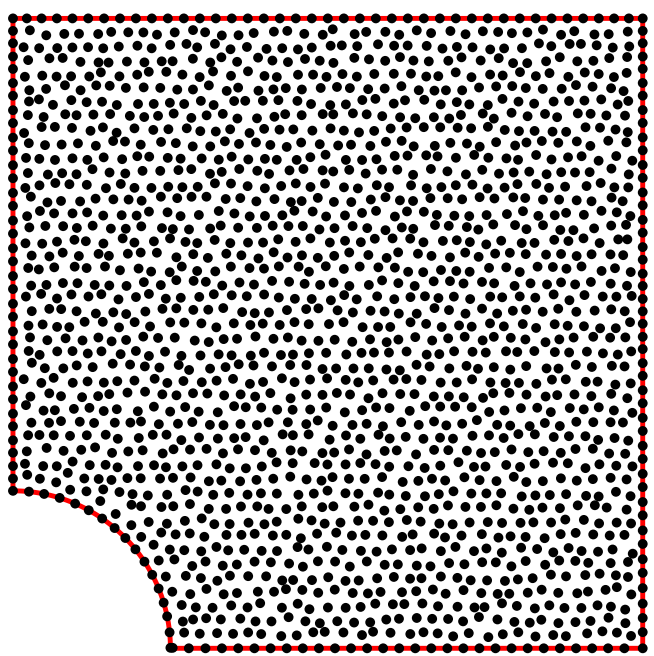

(d)

Figure 13: Nodal discretizations for the elastic plate with hole problem. The first two discretizations (a) and (b), with 456 and 1779 nodes respectively, are generated with the Matlab mesher distmesh. To obtain irregular distributions, the internal nodes are moved by adding a random displacement perturbation whose components are in the range of $[-0.2 h 0.2 h]$ in (c) and (d). 


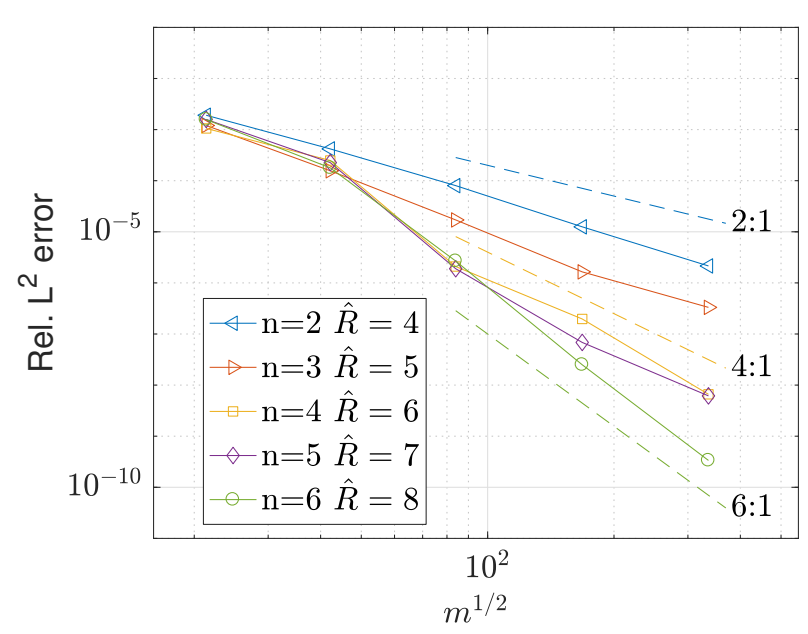

(a) Displacements

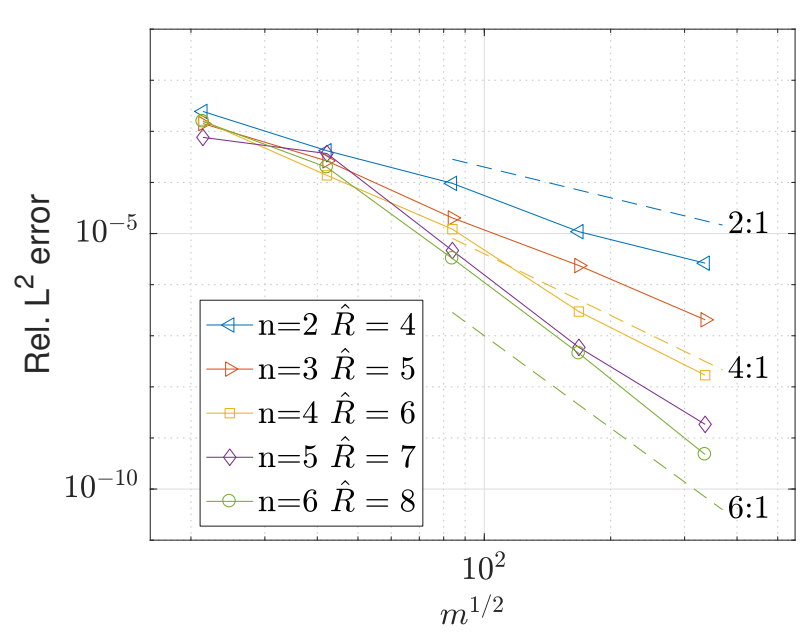

(c) Displacements

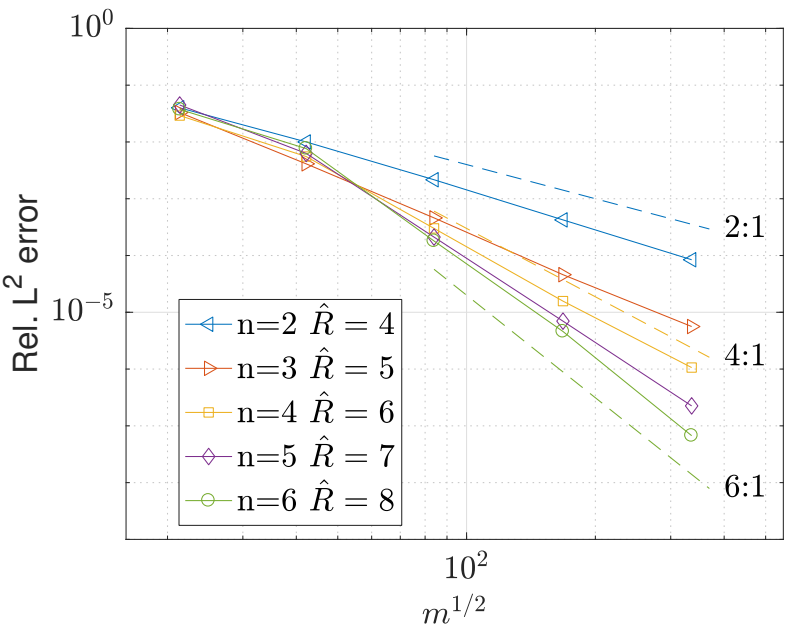

(b) Stress

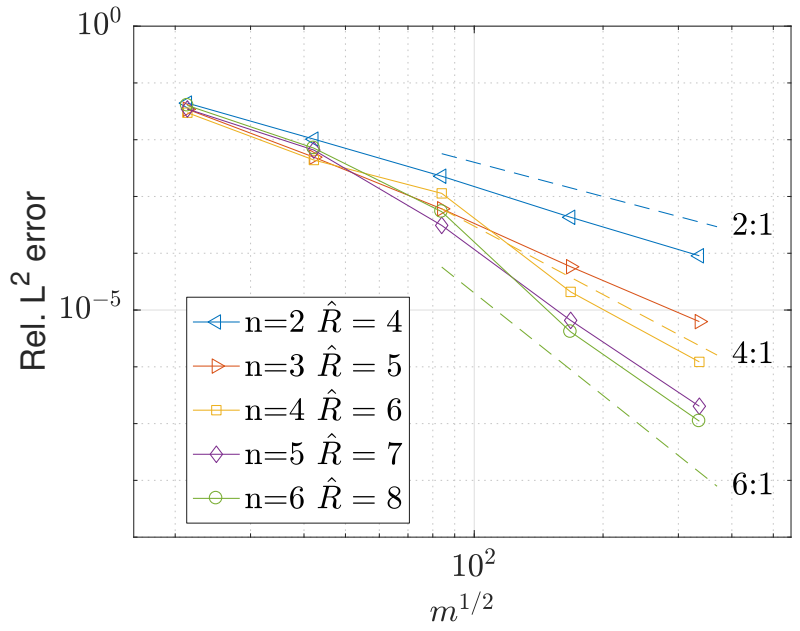

(d) Stress

Figure 14: Convergence of the relative $L^{2}$ error of the displacements and stress fields for the infinite plate with hole problem, solved for different values of $n$ and $p=2$. Regular nodal distributions are considered in (a) and (b), while a perturbation of $0.2 h$ is considered in $(c)$ and $(d)$. 


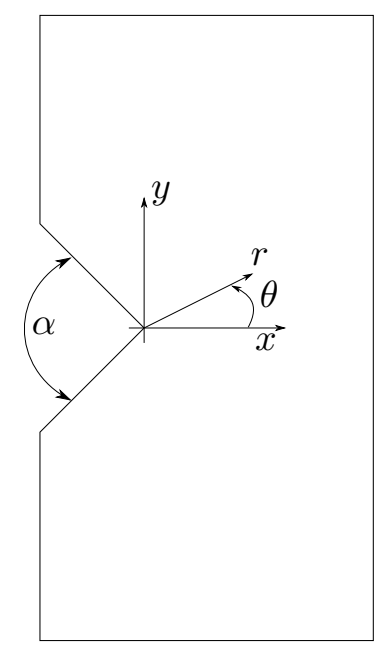

Figure 15: Elastic plate with a re-entrant corner.

point was given in [56] and depends on the particular traction configuration. In this example, we consider a corner angle $\alpha=\pi / 2$ and reproduce the Mode I term of the asymptotic expression of the displacement field, which is given by

$$
\begin{aligned}
& u(r, \theta)=r^{\lambda_{1}} \frac{1}{2 G}\left\{\left[\kappa-Q\left(\lambda_{1}+1\right)\right] \cos \left(\lambda_{1} \theta\right)-\lambda_{1} \cos \left[\left(\lambda_{1}-2\right) \theta\right]\right\}, \\
& v(r, \theta)=r^{\lambda_{1}} \frac{1}{2 G}\left\{\left[\kappa+Q\left(\lambda_{1}+1\right)\right] \sin \left(\lambda_{1} \theta\right)+\lambda_{1} \sin \left[\left(\lambda_{1}-2\right) \theta\right]\right\},
\end{aligned}
$$

where $\lambda_{1}$ is an eigenvalue that determines the order of the singularity and $Q$ is a constant, both depending in general on the corner angle $\alpha$. In particular, for $\alpha=\pi / 2$, their value is given by $\lambda_{1}=0.544483737$ and $Q=0.543075579$. The interested reader is referred to [57] for a more detailed discussion of the general solution. The above displacement field is imposed as an essential boundary condition and the problem is solved assuming a state of plane stress and the material parameters $E=1$ and $\nu=0.3$. The corresponding stress field is given by

$$
\begin{aligned}
& \sigma_{x}(r, \theta)=\lambda_{1} r^{\lambda_{1}-1}\left\{\left[2-Q\left(\lambda_{1}+1\right)\right] \cos \left[\left(\lambda_{1}-1\right) \theta\right]-\left(\lambda_{1}-1\right) \cos \left[\left(\lambda_{1}-3\right) \theta\right]\right\}, \\
& \sigma_{y}(r, \theta)=\lambda_{1} r^{\lambda_{1}-1}\left\{\left[2+Q\left(\lambda_{1}+1\right)\right] \cos \left[\left(\lambda_{1}-1\right) \theta\right]+\left(\lambda_{1}-1\right) \cos \left[\left(\lambda_{1}-3\right) \theta\right]\right\}, \\
& \tau_{x y}(r, \theta)=\lambda_{1} r^{\lambda_{1}-1}\left\{Q\left(\lambda_{1}+1\right) \sin \left[\left(\lambda_{1}-1\right) \theta\right]+\left(\lambda_{1}-1\right) \sin \left[\left(\lambda_{1}-3\right) \theta\right]\right\},
\end{aligned}
$$

and presents a singularity at the corner, which limits the convergence of the numerical solution. In particular, for finite element Galerkin formulations, it is well known that the energy error converges with a rate equal to $\lambda_{1}$, independently of the approximation polynomial order $n$ [57]. As discussed earlier, a theoretical framework for the numerical analysis of collocation methods has 


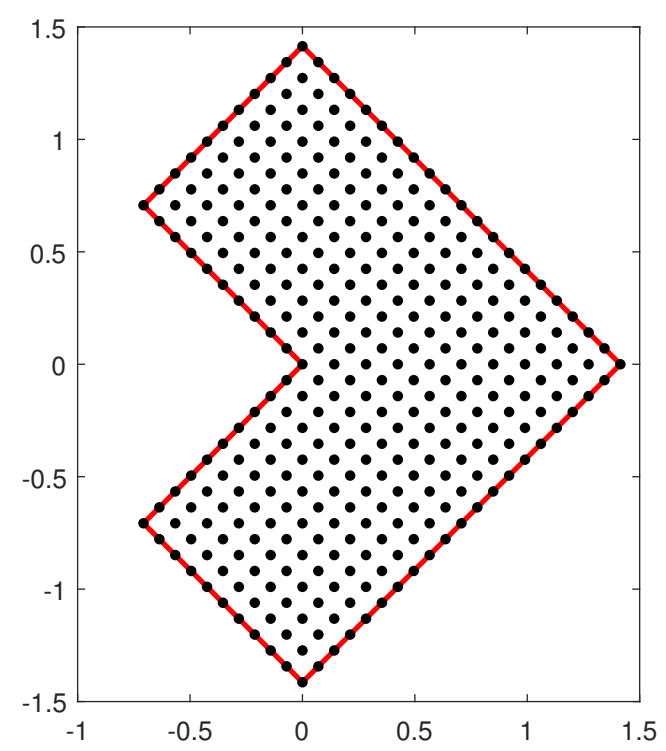

(a)

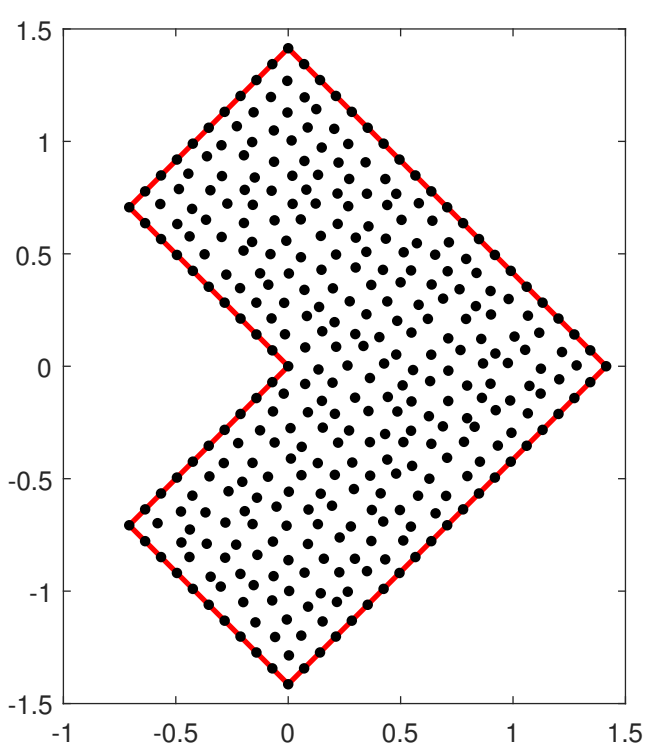

(b)

Figure 16: Nodal discretizations for the L-shaped elastic plate with singularity problem. The discretization in (a) is generated by merging three square tensor product grids. To obtain an irregular distribution, the internal nodes are moved by adding a random perturbation whose components are in the range of $[-0.2 h 0.2 h]$ in (b).

not been completely developed yet. The presence of singularities further complicates the problem and convergence rates are estimated in the literature based on numerical studies only [3,9].

The plate domain is discretized using regular and irregular nodal distributions, as outlined in Fig. 16. In this case, the regular distributions are obtained by merging together three tensor product grids, generated in the unit square, and the irregular ones as for the previous elasticity example.

The relative convergence studies are reported in Fig. 17, where it can be observed how the stress error converges with a rate of $\lambda_{1}$ for both the regular and irregular grid cases, which is in agreement with the theoretical prediction for Galerkin methods. Interestingly, the displacement error converges with a faster rate of $\sim 1.3$.

\subsection{Poisson equation on a domain represented by a NURBS curve}

The numerical results presented in the previous sections showed the accuracy of HOLMES collocation in different applications. Both the acoustic and the infinite plate with hole problems are defined on circular domains (spherical in 3D) and the expected convergence rates are obtained 


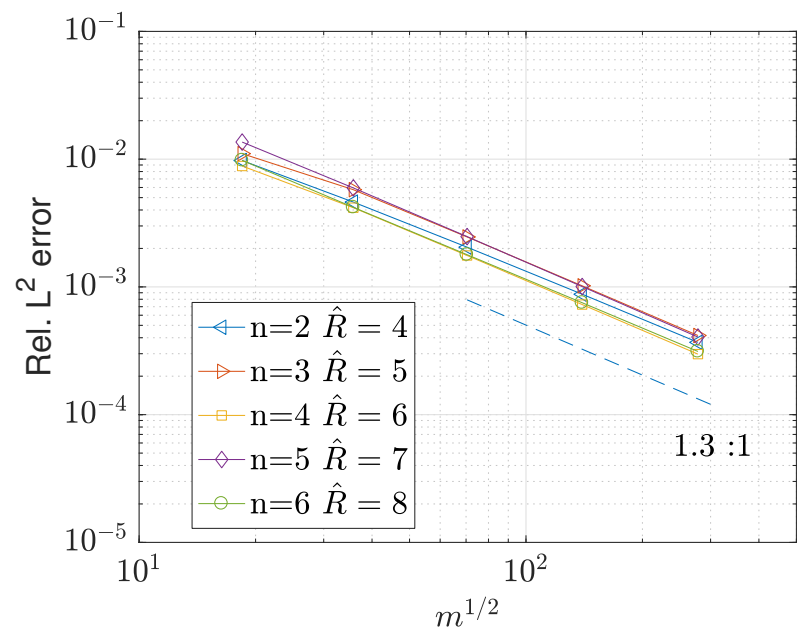

(a) Displacements

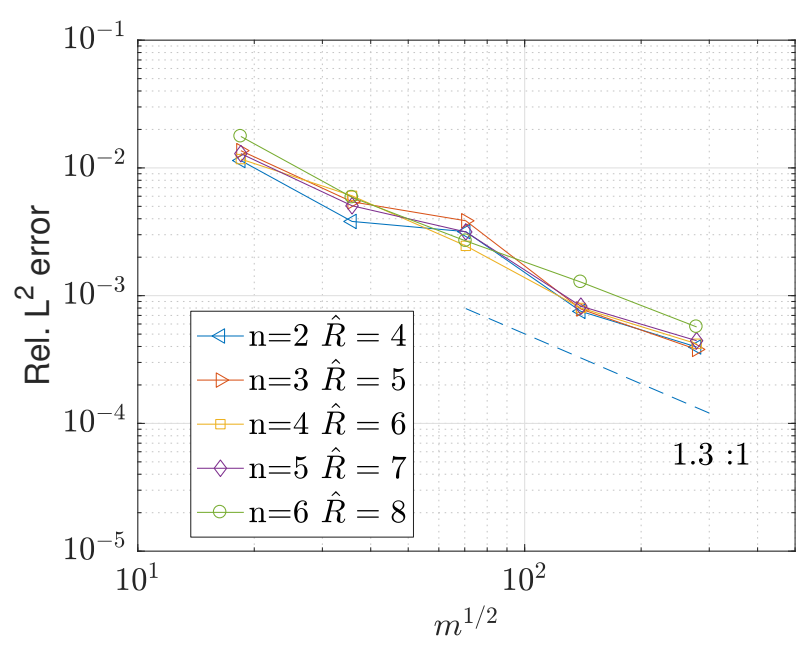

(c) Displacements

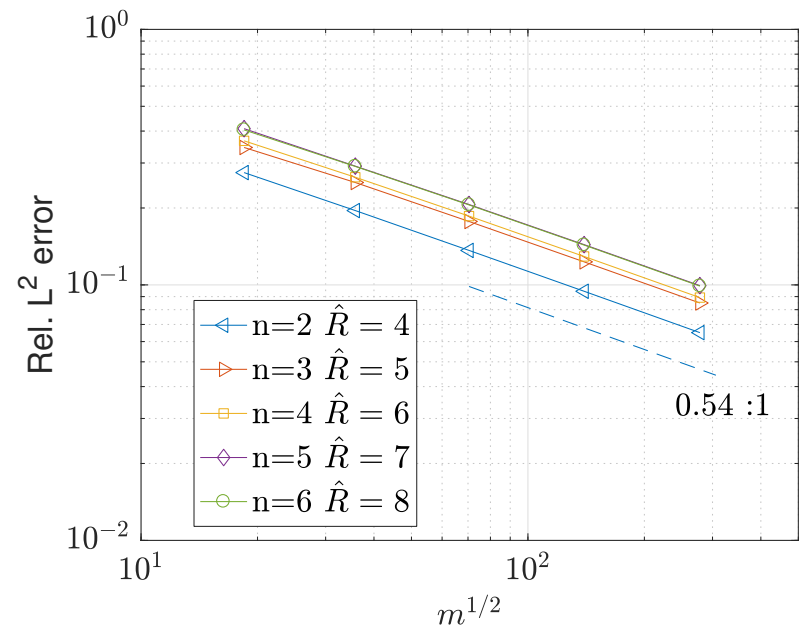

(b) Stress

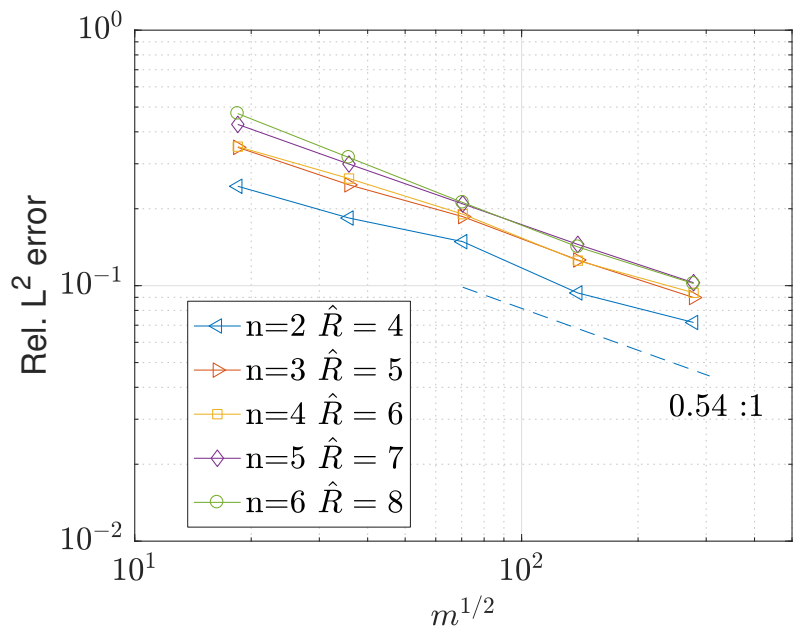

(d) Stress

Figure 17: Convergence of the relative $L^{2}$ error of the displacements and stress fields for the L-shaped elastic plate with singularity problem, solved for different values of $n$ and $p=2$. Regular nodal distributions are considered in (a) and (b), while a perturbation of $0.2 h$ is considered in (c) and (d). 


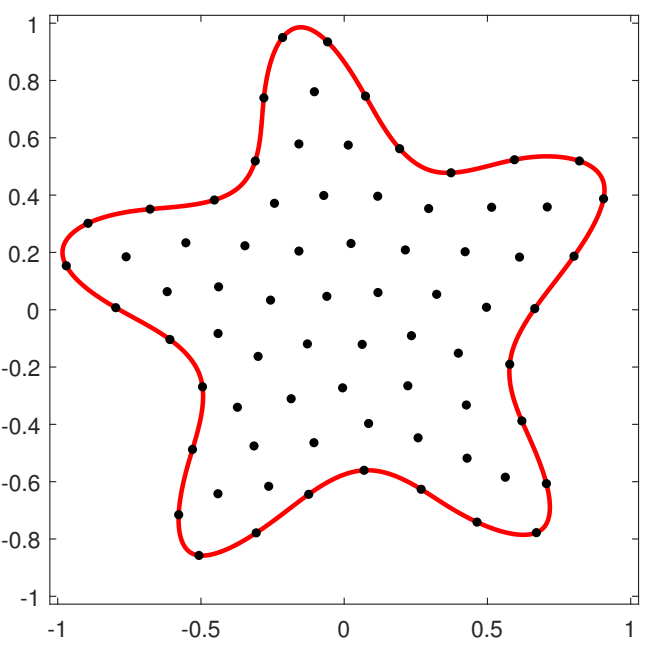

(a) 74 nodes

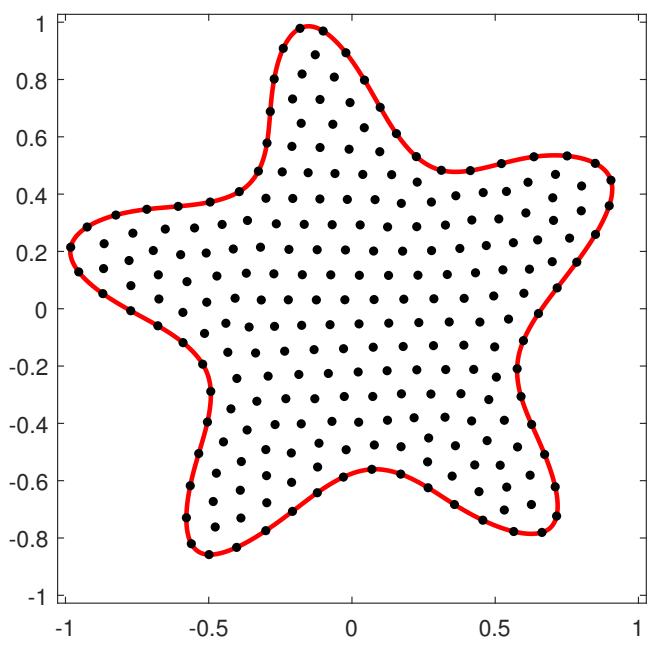

(b) 244 nodes

Figure 18: A star-shaped domain defined by a NURBS curve and two discretizations used for the convergence study.

for HOLMES of different orders. For the acoustic Helmholtz problem with natural boundary conditions, this is not the case of Galerkin formulations integrated on the Delaunay triangulation of the nodes where, even if high-order basis functions are employed, the convergence rate is limited to the value of $2: 1$ given by the geometrical error unless special integration rules are used [53]. On the other hand, imposing the boundary conditions on the exact boundary description is enough for the collocation formulation to converge with the expected rates.

As discussed in the introduction, many efforts have been made over the last few years to seek a tighter integration between $\mathrm{CAD}$ and numerical analysis and, in this context, meshfree collocation can be a possible way to directly approximate PDEs on CAD geometric models [44]. This concept is examined in this section with an example of a star-shaped domain defined by a NURBS curve, which is the standard employed in CAD. The geometry is shown in Fig. 18 and the coordinates of the control points are given in the Appendix.

To implement the collocation procedure, the computational grid is generated as a proof of concept with the FEM mesher distmesh, like in the other examples considered in this work. However, as discussed in [44], specific algorithms can be developed for the automatic grid generation on complex CAD models.

The following Poisson problem is solved

$$
\Delta u(\mathbf{x})=b(\mathbf{x}), \quad x \in \Omega,
$$




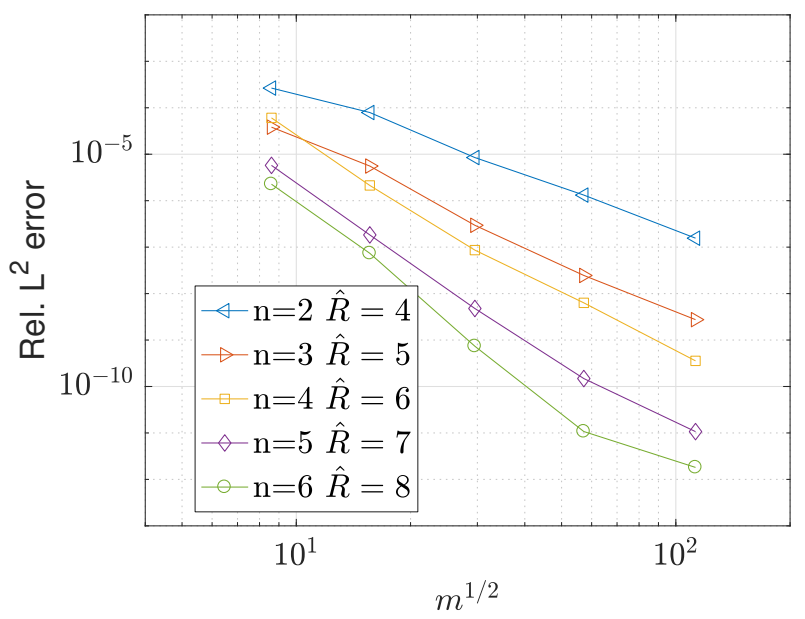

(a) $u_{1}(\mathbf{x})$

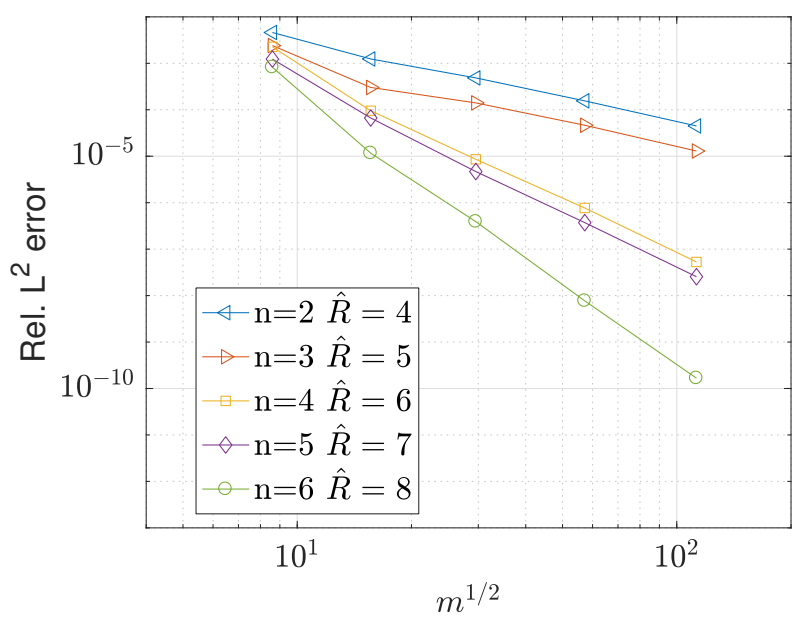

(b) $u_{2}(\mathbf{x})$

Figure 19: Convergence of the relative $L^{2}$ error for a Poisson problem solved on a star shaped domain defined by a NURBS curve for different values of $n$, and $p=2$.

and, as for the 1D case of Section 4.1, source terms and essential boundary conditions are imposed to reproduce the following functions

$$
\begin{aligned}
& u_{1}(\mathbf{x})=\sinh (x) \cos (y) \\
& u_{2}(\mathbf{x})=e^{x^{2}+y^{2}}+\sinh (x) \cos (2 y) .
\end{aligned}
$$

The convergence curves of the relative $L^{2}$ error are reported in Fig. 19, where the expected rates are found in all the cases, with a super-convergent behavior in most of the curves for $u_{1}(\mathbf{x})$. These results confirm how the discretization with HOLMES approximants and the imposition of the boundary conditions directly on the points belonging to the NURBS boundary ensure the correct convergence behavior, allowing a direct integration between the CAD representation and the numerical analysis.

\section{Concluding remarks}

In this work, a collocation framework based on HOLMES approximation schemes was applied to the resolution of PDEs. This approach introduces some remarkable advantages with respect to the Galerkin formulation. A relevant one is that the computational times for assembling the stiffness matrix are reduced since the number of evaluation points of the basis functions and their derivatives is significantly lower and no memory bottlenecks for writing in the matrix occur. In 
addition, by avoiding a background integration grid, a truly meshfree character is obtained, which can be useful for many applications such as problems involving large deformations.

The numerical examples confirmed the validity and the accuracy of the proposed methodology in different applications. They also suggested that, in contrast to the Galerkin formulation, HOLMES collocation converges with a value of $p=2$ for the locality norm, independently of the order $n$. Furthermore, $p=2$ shows more regular convergence trends as compared to higher values of $p$. A theoretical study on the convergence of the method, to support these numerical findings, can be the topic of future research. However, a framework for the numerical analysis of collocation methods is not nearly as mature as for Galerkin methods [2]. The character of the stiffness matrix, which is no longer symmetric and positive definite, is another drawback with respect to Galerkin methods.

This work also showed how the proposed HOLMES collocation procedure allows us to approximate PDEs on domains with a smooth geometric definition, given either by an explicit description or by a NURBS curve, which can be a very useful methodology to integrate CAD and numerical analysis. Therefore, its application to complex 3D models with the development of an automated procedure for the grid generation, such as that used in [44], can also be an interesting topic of future research.

\section{Acknowledgments}

The research of F. Greco has received funding from the European Union's Horizon 2020 research and innovation programme, under the Marie Sklodowska-Curie grant agreement No 792028.

\section{References}

[1] J. A. Cottrell, T. J. R. Hughes, and Y. Bazilevs, Isogeometric analysis: toward integration of CAD and FEA. John Wiley \& Sons, 2009.

[2] F. Auricchio, L. B. Da Veiga, T. J. Hughes, A. Reali, and G. Sangalli, "Isogeometric collocation methods," Mathematical Models and Methods in Applied Sciences, vol. 20, no. 11, pp. 2075-2107, 2010.

[3] D. Schillinger, J. A. Evans, A. Reali, M. A. Scott, and T. J. Hughes, "Isogeometric collocation: Cost comparison with Galerkin methods and extension to adaptive hierarchical NURBS discretizations," Computer Methods in Applied Mechanics and Engineering, vol. 267, pp. 170-232, dec 2013.

[4] A. Reali and T. J. Hughes, "An Introduction to Isogeometric Collocation Methods," in CISM International Centre for Mechanical Sciences, Courses and Lectures, vol. 561, pp. 173-204, 2015. 
[5] R. Kruse, N. Nguyen-Thanh, L. De Lorenzis, and T. J. Hughes, "Isogeometric collocation for large deformation elasticity and frictional contact problems," Computer Methods in Applied Mechanics and Engineering, vol. 296, pp. $73-112,2015$.

[6] H. Gomez, A. Reali, and G. Sangalli, "Accurate, efficient, and (iso)geometrically flexible collocation methods for phase-field models," Journal of Computational Physics, vol. 262, pp. 153-171, 2014.

[7] E. Marino, "Isogeometric collocation for three-dimensional geometrically exact shear-deformable beams," Computer Methods in Applied Mechanics and Engineering, vol. 307, pp. 383-410, 2016.

[8] J. Kiendl, E. Marino, and L. De Lorenzis, "Isogeometric collocation for the Reissner-Mindlin shell problem," Computer Methods in Applied Mechanics and Engineering, vol. 325, pp. 645-665, 2017.

[9] F. Maurin, F. Greco, L. Coox, D. Vandepitte, and W. Desmet, "Isogeometric collocation for Kirchhoff-Love plates and shells," Computer Methods in Applied Mechanics and Engineering, vol. 329, pp. 396-420, 2018.

[10] H. Gomez and L. De Lorenzis, "The variational collocation method," Computer Methods in Applied Mechanics and Engineering, vol. 309, pp. 152-181, 2016.

[11] M. Montardini, G. Sangalli, and L. Tamellini, "Optimal-order isogeometric collocation at Galerkin superconvergent points," Computer Methods in Applied Mechanics and Engineering, vol. 316, pp. 741-757, 2017.

[12] M. A. Scott, T-splines as a design-through-analysis technology. Phd thesis, The University of Texas at Austin, 2011.

[13] X. Wei, Y. J. Zhang, T. J. R. Hughes, and M. A. Scott, "Extended Truncated Hierarchical Catmull-Clark Subdivision," Computer Methods in Applied Mechanics and Engineering, vol. 299, pp. 316-336, 2016.

[14] T. Belytschko, Y. Krongauz, D. Organ, M. Fleming, and P. Krysl, "Meshless methods: An overview and recent developments," Computer Methods in Applied Mechanics and Engineering, vol. 139, no. 1, pp. 3-47, 1996.

[15] A. Huerta, T. Belytschko, S. Fernández-Méndez, and T. Rabczuk, "Meshfree Methods," vol. 1 of Encyclopedia of Computational Mechanics, ch. 10, pp. 279-309, John Wiley \& Sons, Ltd., 2004.

[16] S. Li and W. K. Liu, "Meshfree and particle methods and their applications," Applied Mechanics Reviews, vol. 55, no. 1, pp. 1-34, 2002.

[17] J.-S. Chen and T. Belytschko, "Meshless and Meshfree Methods," Encyclopedia of Applied and Computational Mathematics, pp. 886-894, 2015.

[18] J.-S. Chen, C.-T. Wu, S. Yoon, and Y. You, "A stabilized confirming nodal integration for Galerkin mesh-free methods," International Journal for Numerical Methods in Engineering, vol. 50, pp. 435-466, 2001.

[19] W. Benz, "Smooth Particle Hydrodynamics: A Review," The Numerical Modelling of Nonlinear Stellar Pulsations, pp. 269-288, 1990.

[20] E. J. Kansa, "Multiquadrics-A scattered data approximation scheme with applications to computational fluiddynamics-I surface approximations and partial derivative estimates," Computers and Mathematics with Applications, vol. 19, no. 8-9, pp. 127-145, 1990.

[21] N. R. Aluru, "A point collocation method based on reproducing kernel approximations," International Journal for Numerical Methods in Engineering, vol. 47, no. 6, pp. 1083-1121, 2000.

[22] H. Y. Hu, J. S. Chen, and W. Hu, "Error analysis of collocation method based on reproducing kernel approximation," Numerical Methods for Partial Differential Equations, vol. 27, no. 3, pp. 554-580, 2011. 
[23] Z. H. Yao, M. W. Yuan, and A. H.-D. Cheng, "Radial Basis Function Collocation Method," Computational Mechanics, pp. 219-219, 2007.

[24] X. Zhang, K. Z. Song, M. W. Lu, and X. Liu, "Meshless methods based on collocation with radial basis functions," Computational Mechanics, vol. 26, no. 4, pp. 333-343, 2000.

[25] E. Oñate, S. Idelsohn, O. C. Zienkiewicz, and R. L. Taylor, "A finite point method in computational mechanics. Applications to convective transport and fluid flow," International Journal for Numerical Methods in Engineering, vol. 39, pp. 3839-3866, nov 1996.

[26] S. Garg and M. Pant, "Meshfree Methods: A Comprehensive Review of Applications," International Journal of Computational Methods, vol. 15, no. 4, 2018.

[27] M. Hillman and J. S. Chen, "Performance comparison of nodally integrated galerkin meshfree methods and nodally collocated strong form meshfree methods," Computational Methods in Applied Sciences, vol. 46, pp. 145164, 2018.

[28] N. Sukumar, "Construction of polygonal interpolants: A maximum entropy approach," International Journal for Numerical Methods in Engineering, vol. 61, no. 12, pp. 2159-2181, 2004.

[29] M. Arroyo and M. Ortiz, "Local maximum-entropy approximation schemes: A seamless bridge between finite elements and meshfree methods," International Journal for Numerical Methods in Engineering, vol. 65, no. 13, pp. 2167-2202, 2006.

[30] C. J. Cyron, M. Arroyo, and M. Ortiz, "Smooth, second order, non-negative meshfree approximants selected by maximum entropy," International Journal for Numerical Methods in Engineering, vol. 79, no. 13, pp. 1605-1632, 2009.

[31] A. Rosolen, D. Millán, and M. Arroyo, "Second-order convex maximum entropy approximants with applications to high-order PDE," International Journal for Numerical Methods in Engineering, vol. 94, no. 2, pp. 150-182, 2013.

[32] A. Bompadre, L. E. Perotti, C. J. Cyron, and M. Ortiz, "Convergent meshfree approximation schemes of arbitrary order and smoothness," Computer Methods in Applied Mechanics and Engineering, vol. 221, pp. 83103, 2012.

[33] D. Wang and P. Chen, "Quasi-convex reproducing kernel meshfree method," Computational Mechanics, pp. 689709, 2014.

[34] A. Ortiz, M. A. Puso, and N. Sukumar, "Maximum-entropy meshfree method for compressible and nearincompressible elasticity," Computer Methods in Applied Mechanics and Engineering, vol. 199, no. 25, pp. 1859$1871,2010$.

[35] F. Amiri, D. Millán, Y. Shen, T. Rabczuk, and M. Arroyo, "Phase-field modeling of fracture in linear thin shells," Theoretical and Applied Fracture Mechanics, vol. 69, pp. 102-109, 2014.

[36] D. Millán, A. Rosolen, and M. Arroyo, "Thin shell analysis from scattered points with maximum-entropy approximants," International Journal for Numerical Methods in Engineering, vol. 85, pp. 723-751, feb 2011.

[37] C. J. Cyron, K. Nissen, V. Gravemeier, and W. A. Wall, "Stable meshfree methods in fluid mechanics based on Green's functions," Computational Mechanics, vol. 46, no. 2, pp. 287-300, 2010.

[38] F. Greco, L. Coox, and W. Desmet, "Maximum-entropy methods for time-harmonic acoustics," Computer 
Methods in Applied Mechanics and Engineering, vol. 306, pp. 1-18, 2016.

[39] F. Perazzo and F. Marchant, "Análisis comparativo entre funciones de forma maxent y de mínimos cuadrados ponderados en un método sin malla de colocación," Revista Internacional de Metodos Numericos para Calculo y Diseno en Ingenieria, vol. 33, pp. 290-298, jul 2017.

[40] L. Fan, W. M. Coombs, and C. E. Augarde, "The point collocation method with a local maximum entropy approach," Computers and Structures, vol. 201, pp. 1-14, 2018.

[41] L. Fan, Adaptive meshless point collocation methods: investigation and application to geometrically non-linear solid mechanics. Phd thesis, University of Durham, 2019.

[42] D. Wang, J. Wang, and J. Wu, "Superconvergent gradient smoothing meshfree collocation method," Computer Methods in Applied Mechanics and Engineering, vol. 340, pp. 728-766, 2018.

[43] H.-Y. Hu, C.-K. Lai, and J.-S. Chen, "A study on convergence and complexity of reproducing kernel collocation method," Interaction and multiscale mechanics, vol. 2, no. 3, pp. 295-319, 2009.

[44] S. M. Mirfatah, B. Boroomand, and E. Soleimanifar, "On the solution of 3D problems in physics: From the geometry definition in CAD to the solution by a meshless method," Journal of Computational Physics, vol. 393, pp. 351-374, 2019.

[45] L. Zhang, T. Cui, H. Liu, and Others, "A set of symmetric quadrature rules on triangles and tetrahedra," J. Comput. Math, vol. 27, no. 1, pp. 89-96, 2009.

[46] C. Peco, D. Millán, A. Rosolen, and M. Arroyo, "Efficient implementation of Galerkin meshfree methods for large-scale problems with an emphasis on maximum entropy approximants," Computers $\mathcal{G}$ Structures, vol. 150, pp. 52-62, 2015.

[47] E. T. Jaynes, "Information theory and statistical mechanics. II," Physical Review, vol. 108, no. 2, pp. 171-190, 1957.

[48] F. Greco and N. Sukumar, "Derivatives of maximum-entropy basis functions on the boundary: Theory and computations," International Journal for Numerical Methods in Engineering, vol. 94, no. 12, pp. 1123-1149, 2013.

[49] I. Babuška and J. M. Melenk, "The partition of unity method," International Journal for Numerical Methods in Engineering, vol. 40, no. 4, pp. 727-758, 1997.

[50] D. González, E. Cueto, and M. Doblaré, "A higher order method based on local maximum entropy approximation," International Journal for Numerical Methods in Engineering, vol. 83, no. 6, pp. n/a-n/a, 2010.

[51] N. Sukumar and R. W. Wright, "Overview and construction of meshfree basis functions: from moving least squares to entropy approximants," International Journal for Numerical Methods in Engineering, vol. 70, pp. 181205, apr 2007.

[52] A. Bompadre, B. Schmidt, and M. Ortiz, "Convergence analysis of meshfree approximation schemes," SIAM Journal on Numerical Analysis, vol. 50, no. 3, pp. 1344-1366, 2012.

[53] F. Greco, L. Coox, F. Maurin, and W. Desmet, "NURBS-enhanced maximum-entropy schemes," Computer Methods in Applied Mechanics and Engineering, vol. 317, pp. 580-597, 2017.

[54] P. Persson and G. Strang, "A Simple Mesh Generator in MATLAB," SIAM Review, vol. 46, no. 2, pp. 329-345, 2004. 
[55] S. Timoshenko, "Theory Of Elasticity. 1st. Ed.," 1934.

[56] M. L. Williams and C. Pasadena, "Stress singularities resulting from various boundary conditions in angular corners of plate in extension," Journal of Applied Mechanics, vol. 19, pp. 526-534, 1952.

[57] B. A. Szabó and I. Babuška, An Introduction to Finite Element Analysis. 2011. 


\section{Appendix}

The NURBS curve representing the star domain of Section 4.4 is a periodic cubic curve obtained from an equi-spaced knot vector and 20 control points, whose coordinates and weights are given below.

\begin{tabular}{|c|c|c|c|}
\hline Index i & $x_{i}$ & $y_{i}$ & $w_{i}$ \\
\hline 1 & -0.315646 & -1.085310 & 1.515248 \\
\hline 2 & 0.091382 & -0.554301 & 1.180104 \\
\hline 3 & 0.471145 & -0.906028 & 1.275062 \\
\hline 4 & 0.658987 & -0.724081 & 0.805530 \\
\hline 5 & 0.717332 & -0.497103 & 1.085006 \\
\hline 6 & 0.612211 & -0.207935 & 1.204812 \\
\hline 7 & 1.225410 & 0.199055 & 1.602287 \\
\hline 8 & 1.317630 & 0.653927 & 1.287513 \\
\hline 9 & 0.781819 & 0.724652 & 1.279621 \\
\hline 10 & 0.308763 & 0.447180 & 1.146973 \\
\hline 11 & 0.104881 & 1.024695 & 1.371792 \\
\hline 12 & -0.139101 & 0.885485 & 0.747637 \\
\hline 13 & -0.328176 & 0.819813 & 1.104964 \\
\hline 14 & -0.310237 & 0.389074 & 1.089473 \\
\hline 15 & -1.013978 & 0.505792 & 1.398823 \\
\hline 16 & -0.948944 & 0.187873 & 0.793557 \\
\hline 17 & -1.055274 & -0.025958 & 1.393367 \\
\hline 18 & -0.498210 & -0.237582 & 1.208597 \\
\hline 19 & -0.769951 & -0.761041 & 1.349239 \\
\hline 20 & -0.667820 & -1.073769 & 1.081645 \\
\hline
\end{tabular}

